九州大学学術情報リポジトリ

Kyushu University Institutional Repository

\title{
Multi-poly-Bernoulli numbers and related zeta functions
}

Kaneko, Masanobu

Faculty of Mathematics, Kyushu University : Professor

Tsumura, Hirofumi

Department of Mathematics and Information Sciences, Tokyo Metropolitan University

http://hdl. handle. net/2324/4753048

出版情報: Nagoya Mathematical Journal. 232, pp. 19-54，2017-05-08. 名古屋大学理学部数学教室 バージョン：

権利関係 : 


\title{
Multi-poly-Bernoulli numbers and related zeta functions
}

\author{
Masanobu Kaneko*and Hirofumi Tsumura
}

\begin{abstract}
We construct and study a certain zeta function which interpolates multi-poly-Bernoulli numbers at non-positive integers and whose values at positive integers are linear combinations of multiple zeta values. This function can be regarded as the one to be paired up with the $\xi$-function defined by Arakawa and the first-named author. We show that both are closely related to the multiple zeta functions. Further we define multi-indexed poly-Bernoulli numbers, and generalize the duality formulas for poly-Bernoulli numbers by introducing more general zeta functions.
\end{abstract}

\section{Introduction}

In this paper, we investigate the function defined by

$$
\eta\left(k_{1}, \ldots, k_{r} ; s\right)=\frac{1}{\Gamma(s)} \int_{0}^{\infty} t^{s-1} \frac{\operatorname{Li}_{k_{1}, \ldots, k_{r}}\left(1-e^{t}\right)}{1-e^{t}} d t
$$

and its generalizations, in connection with multi-poly-Bernoulli numbers and multiple zeta values (we shall give the precise definitions later in $\S 2$ ). This function can be viewed as a twin sibling of the function $\xi\left(k_{1}, \ldots, k_{r} ; s\right)$,

$$
\xi\left(k_{1}, \ldots, k_{r} ; s\right)=\frac{1}{\Gamma(s)} \int_{0}^{\infty} t^{s-1} \frac{\operatorname{Li}_{k_{1}, \ldots, k_{r}}\left(1-e^{-t}\right)}{e^{t}-1} d t,
$$

which was introduced and studied in [4]. The present paper may constitute a natural continuation of the work [4].

To explain our results in some detail, we first give an overview of the necessary background. For an integer $k \in \mathbb{Z}$, two types of poly-Bernoulli numbers $\left\{B_{n}^{(k)}\right\}$ and $\left\{C_{n}^{(k)}\right\}$ are defined as follows (see Kaneko [20] and Arakawa-Kaneko [4], also Arakawa-Ibukiyama-Kaneko [3]):

$$
\begin{aligned}
& \frac{\operatorname{Li}_{k}\left(1-e^{-t}\right)}{1-e^{-t}}=\sum_{n=0}^{\infty} B_{n}^{(k)} \frac{t^{n}}{n !}, \\
& \frac{\operatorname{Li}_{k}\left(1-e^{-t}\right)}{e^{t}-1}=\sum_{n=0}^{\infty} C_{n}^{(k)} \frac{t^{n}}{n !},
\end{aligned}
$$

${ }^{*}$ This work was supported by Japan Society for the Promotion of Science, Grant-in-Aid for Scientific Research (B) 23340010.

2010 Mathematics Subject Classification: Primary 11B68; Secondary 11M32

Key words: multi-poly-Bernoulli number, multiple zeta value, Arakawa-Kaneko zeta function 
where $\operatorname{Li}_{k}(z)$ is the polylogarithm function defined by

$$
\operatorname{Li}_{k}(z)=\sum_{m=1}^{\infty} \frac{z^{m}}{m^{k}} \quad(|z|<1)
$$

Since $\operatorname{Li}_{1}(z)=-\log (1-z)$, we see that $B_{n}^{(1)}\left(\right.$ resp. $\left.C_{n}^{(1)}\right)$ coincides with the ordinary Bernoulli number $B_{n}$ defined by

$$
\frac{t e^{t}}{e^{t}-1}=\sum_{n=0}^{\infty} B_{n} \frac{t^{n}}{n !} \quad\left(\text { resp. } \frac{t}{e^{t}-1}=\sum_{n=0}^{\infty} B_{n} \frac{t^{n}}{n !}\right) .
$$

A number of formulas, including closed formulas of $B_{n}^{(k)}$ and $C_{n}^{(k)}$ in terms of the Stirling numbers of the second kind as well as the duality formulas

$$
\begin{aligned}
& B_{n}^{(-k)}=B_{k}^{(-n)}, \\
& C_{n}^{(-k-1)}=C_{k}^{(-n-1)}
\end{aligned}
$$

that hold for $k, n \in \mathbb{Z}_{\geq 0}$, have been established (see [20, Theorems 1 and 2] and [21, §2]). We also mention that Brewbaker [9] gave a purely combinatorial interpretation of the number $B_{n}^{(-k)}$ of negative upper index as the number of 'Lonesum-matrices' with $n$ rows and $k$ columns.

A multiple version of $B_{n}^{(k)}$ is defined in [4, p. 202, Remarks (ii)] by

$$
\frac{\operatorname{Li}_{k_{1}, \ldots, k_{r}}\left(1-e^{-t}\right)}{\left(1-e^{-t}\right)^{r}}=\sum_{n=0}^{\infty} \mathbb{B}_{n}^{\left(k_{1}, \ldots, k_{r}\right)} \frac{t^{n}}{n !} \quad\left(k_{1}, \ldots, k_{r} \in \mathbb{Z}\right),
$$

where

$$
\operatorname{Li}_{k_{1}, \ldots, k_{r}}(z)=\sum_{1 \leq m_{1}<\cdots<m_{r}} \frac{z^{m_{r}}}{m_{1}^{k_{1}} m_{2}^{k_{2}} \cdots m_{r}^{k_{r}}}
$$

is the multiple polylogarithm. Hamahata and Masubuchi $[14,15]$ investigated some properties of $\mathbb{B}_{n}^{\left(k_{1}, \ldots, k_{r}\right)}$, and gave several generalizations of the known results in the single-index case. Based on this research, Bayad and Hamahata [8] further studied these numbers. Furusho [12, p. 269] also refers to (1.8).

More recently, Imatomi, Takeda and the first-named author [18] defined and studied another type of multi-poly-Bernoulli numbers given by

$$
\begin{aligned}
& \frac{\operatorname{Li}_{k_{1}, \ldots, k_{r}}\left(1-e^{-t}\right)}{1-e^{-t}}=\sum_{n=0}^{\infty} B_{n}^{\left(k_{1}, \ldots, k_{r}\right)} \frac{t^{n}}{n !}, \\
& \frac{\operatorname{Li}_{k_{1}, \ldots, k_{r}}\left(1-e^{-t}\right)}{e^{t}-1}=\sum_{n=0}^{\infty} C_{n}^{\left(k_{1}, \ldots, k_{r}\right)} \frac{t^{n}}{n !}
\end{aligned}
$$

for $k_{1}, \ldots, k_{r} \in \mathbb{Z}$. They proved several formulas for $B_{n}^{\left(k_{1}, \ldots, k_{r}\right)}$ and $C_{n}^{\left(k_{1}, \ldots, k_{r}\right)}$, and further gave an important relation between $C_{p-2}^{\left(k_{1}, \ldots, k_{r}\right)}$ and the 'finite multiple zeta value', that is,

$$
\sum_{1 \leq m_{1}<\cdots<m_{r}<p} \frac{1}{m_{1}^{k_{1}} \cdots m_{r}^{k_{r}}} \equiv-C_{p-2}^{\left(k_{1}, \ldots, k_{r-1}, k_{r}-1\right)} \bmod p
$$


for any prime number $p$.

The function (1.2) for $k_{1}, \ldots, k_{r} \in \mathbb{Z}_{\geq 1}$ can be analytically continued to an entire function of the complex variable $s \in \mathbb{C}$ ([4, Sections 3 and 4]). The particular case $r=k=1$ gives $\xi(1 ; s)=s \zeta(s+1)$. Hence $\xi\left(k_{1}, \ldots, k_{r} ; s\right)$ can be regarded as a multi-indexed zeta function. It is shown in [4] that the values at non-positive integers of $\xi(k ; s)$ interpolate poly-Bernoulli numbers $C_{m}^{(k)}$,

$$
\xi(k ;-m)=(-1)^{m} C_{m}^{(k)}
$$

for $k \in \mathbb{Z}_{\geq 1}$ and $m \in \mathbb{Z}_{\geq 0}$. And also by investigating $\xi\left(k_{1}, \ldots, k_{r} ; s\right)$ and its values at positive integer arguments, one produces many relations among multiple zeta values defined by

$$
\zeta\left(l_{1}, \ldots, l_{r}\right)=\sum_{1 \leq m_{1}<\cdots<m_{r}} \frac{1}{m_{1}^{l_{1}} \cdots m_{r}^{l_{r}}}\left(=\operatorname{Li}_{l_{1}, \ldots, l_{r}}(1)\right)
$$

for $l_{1}, \ldots, l_{r} \in \mathbb{Z}_{\geq 1}$ with $l_{r} \geq 2([4$, Corollary 11$])$.

Recently, further properties of $\xi\left(k_{1}, \ldots, k_{r} ; s\right)$ and related results have been given by several authors (see, for example, Bayad-Hamahata [6, 7], Coppo-Candelpergher [10], Sasaki [28], and Young [31]).

In this paper, we conduct a basic study of the function (1.1) and relate it to the multi-polyBernoulli numbers $B_{n}^{\left(k_{1}, \ldots, k_{r}\right)}$ as well as multiple zeta (or 'zeta-star') values. Note that the only difference in both definitions (1.1) and (1.2) is, up to sign, the arguments $1-e^{t}$ and $1-e^{-t}$ of $\mathrm{Li}_{k_{1}, \ldots, k_{r}}(z)$ in the integrands. One sees in the main body of the paper a remarkable contrast between ' $B$-type' poly-Bernoulli numbers and those of ' $C$-type', and between multiple zeta and zeta-star values. We further investigate the case of non-positive indices $k_{i}$ in connection with a yet more generalized 'multi-indexed' poly-Bernoulli number.

The paper is organized as follows. In $\S 2$, we give the analytic continuation of $\eta\left(k_{1}, \ldots, k_{r} ; s\right)$ in the case of positive indices, and formulas for values at integer arguments (Theorems 2.3 and 2.5). In $\S 3$, we study relations between two functions $\eta\left(k_{1}, \ldots, k_{r} ; s\right)$ and $\xi\left(k_{1}, \ldots, k_{r} ; s\right)$ (Proposition 3.2), as well as relations with the single variable multiple zeta function (Definition 3.1 and Theorem 3.6). We turn in $\S 4$ to the study of $\eta\left(k_{1}, \ldots, k_{r} ; s\right)$ in the negative index case and give a certain duality formula for $B_{m}^{\left(-k_{1}, \ldots,-k_{r}\right)}$ (Definition 4.3 and Theorems 4.4 and 4.7). We carry forward the study of negative index case in $\S 5$ and define the 'multi-indexed' polyBernoulli numbers $\left\{B_{m_{1}, \ldots, m_{r}}^{\left(k_{1}, \ldots, k_{r}\right),(d)}\right\}$ for $\left(k_{1}, \ldots, k_{r}\right) \in \mathbb{Z}^{r},\left(m_{1}, \ldots, m_{r}\right) \in \mathbb{Z}_{\geq 0}^{r}$ and $d \in\{1, \ldots, r\}$ (Definition 5.1), which include (1.8) and (1.10) as special cases. We prove the 'multi-indexed' duality formula for them in the case $d=r$ (Theorem 5.4).

\section{The function $\eta\left(k_{1}, \ldots, k_{r} ; s\right)$ for positive indices and its values at integers}

\subsection{Analytic continuation and the values at non-positive integers}

We start with the definition in the case of positive indices.

Definition 2.1. For positive integers $k_{1}, \ldots, k_{r} \in \mathbb{Z}_{\geq 1}$, let

$$
\eta\left(k_{1}, \ldots, k_{r} ; s\right)=\frac{1}{\Gamma(s)} \int_{0}^{\infty} t^{s-1} \frac{\operatorname{Li}_{k_{1}, \ldots, k_{r}}\left(1-e^{t}\right)}{1-e^{t}} d t
$$


for $s \in \mathbb{C}$ with $\operatorname{Re}(s)>1-r$, where $\Gamma(s)$ is the gamma function. When $r=1$, we often denote $\eta(k ; s)$ by $\eta_{k}(s)$.

The integral on the right-hand side converges absolutely in the domain $\operatorname{Re}(s)>1-r$, as is seen from the following lemma.

Lemma 2.2. (i) For $k_{1}, \ldots, k_{r} \in \mathbb{Z}_{\geq 1}$, the function $\operatorname{Li}_{k_{1}, \ldots, k_{r}}\left(1-e^{t}\right)$ is holomorphic for $t \in \mathbb{C}$ with $|\operatorname{Im}(t)|<\pi$.

(ii) For $k_{1}, \ldots, k_{r} \in \mathbb{Z}_{\geq 1}$ and $t \in \mathbb{R}_{>0}$, we have the estimates

$$
\operatorname{Li}_{k_{1}, \ldots, k_{r}}\left(1-e^{t}\right)=O\left(t^{r}\right) \quad(t \rightarrow 0)
$$

and

$$
\operatorname{Li}_{k_{1}, \ldots, k_{r}}\left(1-e^{t}\right)=O\left(t^{k_{1}+\cdots+k_{r}}\right) \quad(t \rightarrow \infty) .
$$

Proof. As is well-known, we can regard the function $\operatorname{Li}_{k_{1}, \ldots, k_{r}}(z)$ as a single-valued holomorphic function in the simply connected domain $\mathbb{C} \backslash[1, \infty)$, via the process of iterated integration starting with $\operatorname{Li}_{1}(z)=\int_{0}^{z} d z /(1-z)$. Noting that $1-e^{t} \in[1, \infty)$ is equivalent to $\operatorname{Im}(t)=(2 j+1) \pi$ for some $j \in \mathbb{Z}$, we have the assertion (i).

The estimate (2.1) is clear from the definition of $\operatorname{Li}_{k_{1}, \ldots, k_{r}}(z)$, because its Taylor series at $z=0$ starts with the term $z^{r} / 1^{k_{1}} \cdots r^{k_{r}}$. As for (2.2), we proceed by induction on the 'weight' $k_{1}+\cdots+k_{r}$ as follows by using the formula

$$
\frac{d}{d z} \operatorname{Li}_{k_{1}, \ldots, k_{r}}(z)= \begin{cases}\frac{1}{z} \operatorname{Li}_{k_{1}, \ldots, k_{r-1}, k_{r}-1}(z) & \left(k_{r}>1\right) \\ \frac{1}{1-z} \mathrm{Li}_{k_{1}, \ldots, k_{r-1}}(z) & \left(k_{r}=1\right),\end{cases}
$$

which is easy to derive and is the basis of the analytic continuation of $\operatorname{Li}_{k_{1}, \ldots, k_{r}}(z)$ mentioned above. If $r=k_{1}=1$, then we have $\operatorname{Li}_{1}\left(1-e^{t}\right)=-t$ and the desired estimate holds. Suppose the weight $k$ is larger than 1 and the assertion holds for any weight less than $k$. If $k_{r}>1$, then by $(2.3)$ we have

$$
\begin{aligned}
\left|\operatorname{Li}_{k_{1}, \ldots, k_{r}}\left(1-e^{t}\right)\right| & =\left|\int_{0}^{1-e^{t}} \frac{\operatorname{Li}_{k_{1}, \ldots, k_{r}-1}(u)}{u} d u\right| \\
& =\left|\int_{0}^{t} \frac{1}{1-e^{v}} \operatorname{Li}_{k_{1}, \ldots, k_{r}-1}\left(1-e^{v}\right)\left(-e^{v}\right) d v\right| \quad\left(u:=1-e^{v}\right) \\
& \leq \int_{0}^{\varepsilon}\left|e^{v} \frac{\operatorname{Li}_{k_{1}, \ldots, k_{r}-1}\left(1-e^{v}\right)}{e^{v}-1}\right| d v+\int_{\varepsilon}^{t}\left|\frac{e^{v}}{e^{v}-1} \operatorname{Li}_{k_{1}, \ldots, k_{r}-1}\left(1-e^{v}\right)\right| d v
\end{aligned}
$$

for small $\varepsilon>0$. The former integral is $O(1)$ because the integrand is continuous on $[0, \varepsilon]$. On the other hand, by induction hypothesis, the integrand of the latter integral is $O\left(v^{k_{1}+\cdots+k_{r}-1}\right)$ as $v \rightarrow \infty$. Therefore the latter integral is $O\left(t^{k_{1}+\cdots+k_{r}}\right)$ as $t \rightarrow \infty$. The case of $k_{r}=1$ is similarly proved also by using (2.3), and is omitted here.

We now show that the function $\eta\left(k_{1}, \ldots, k_{r} ; s\right)$ can be analytically continued to an entire function, and interpolates multi-poly-Bernoulli numbers $B_{m}^{\left(k_{1}, \ldots, k_{r}\right)}$ at non-positive integer arguments. 
Theorem 2.3. For positive integers $k_{1}, \ldots, k_{r} \in \mathbb{Z}_{\geq 1}$, the function $\eta\left(k_{1}, \ldots, k_{r} ; s\right)$ can be analytically continued to an entire function on the whole complex plane. And the values of $\eta\left(k_{1}, \ldots, k_{r} ; s\right)$ at non-positive integers are given by

$$
\eta\left(k_{1}, \ldots, k_{r} ;-m\right)=B_{m}^{\left(k_{1}, \ldots, k_{r}\right)} \quad\left(m \in \mathbb{Z}_{\geq 0}\right) .
$$

In particular, $\eta_{k}(-m)=B_{m}^{(k)}$ for $k \in \mathbb{Z}_{\geq 1}$ and $m \in \mathbb{Z}_{\geq 0}$.

Proof. In order to prove this theorem, we adopt here the method of contour integral representation (see, for example, [30, Theorem 4.2]). Let $\mathcal{C}$ be the standard contour, namely the path consisting of the positive real axis from the infinity to (sufficiently small) $\varepsilon$ ('top side'), a counter clockwise circle $C_{\varepsilon}$ around the origin of radius $\varepsilon$, and the positive real axis from $\varepsilon$ to the infinity ('bottom side'). Let

$$
\begin{aligned}
H\left(k_{1}, \ldots, k_{r} ; s\right) & =\int_{\mathcal{C}} t^{s-1} \frac{\operatorname{Li}_{k_{1}, \ldots, k_{r}}\left(1-e^{t}\right)}{1-e^{t}} d t \\
& =\left(e^{2 \pi i s}-1\right) \int_{\varepsilon}^{\infty} t^{s-1} \frac{\operatorname{Li}_{k_{1}, \ldots, k_{r}}\left(1-e^{t}\right)}{1-e^{t}} d t+\int_{C_{\varepsilon}} t^{s-1} \frac{\operatorname{Li}_{k_{1}, \ldots, k_{r}}\left(1-e^{t}\right)}{1-e^{t}} d t .
\end{aligned}
$$

It follows from Lemma 2.2 that $H\left(k_{1}, \ldots, k_{r} ; s\right)$ is entire, because the integrand has no singularity on $\mathcal{C}$ and the contour integral is absolutely convergent for all $s \in \mathbb{C}$. Suppose $\operatorname{Re}(s)>1-r$. The last integral tends to 0 as $\varepsilon \rightarrow 0$. Hence

$$
\eta\left(k_{1}, \ldots, k_{r} ; s\right)=\frac{1}{\left(e^{2 \pi i s}-1\right) \Gamma(s)} H\left(k_{1}, \ldots, k_{r} ; s\right),
$$

which can be analytically continued to $\mathbb{C}$, and is entire. In fact $\eta\left(k_{1}, \ldots, k_{r} ; s\right)$ is holomorphic for $\operatorname{Re}(s)>0$, hence has no singularity at any positive integer. Set $s=-m \in \mathbb{Z}_{\leq 0}$. Then, by $(1.10)$,

$$
\begin{aligned}
\eta\left(k_{1}, \ldots, k_{r} ;-m\right) & =\frac{(-1)^{m} m !}{2 \pi i} H\left(k_{1}, \ldots, k_{r} ;-m\right) \\
& =\frac{(-1)^{m} m !}{2 \pi i} \int_{C_{\varepsilon}} t^{-m-1} \sum_{n=0}^{\infty} B_{n}^{\left(k_{1}, \ldots, k_{r}\right)} \frac{(-t)^{n}}{n !} d t=B_{m}^{\left(k_{1}, \ldots, k_{r}\right)} .
\end{aligned}
$$

This completes the proof.

Remark 2.4. Using the same method as above or the method used in [4], we can establish the analytic continuation of $\xi\left(k_{1}, \ldots, k_{r} ; s\right)$ to an entire function, and see that

$$
\xi\left(k_{1}, \ldots, k_{r} ;-m\right)=(-1)^{m} C_{m}^{\left(k_{1}, \ldots, k_{r}\right)} \quad\left(m \in \mathbb{Z}_{\geq 0}\right)
$$

for $k_{1}, \ldots, k_{r} \in \mathbb{Z}_{\geq 1}$, which is a multiple version of (1.13).

\subsection{Values at positive integers}

About the values at positive integer arguments, we prove formulas for both $\xi\left(k_{1}, \ldots, k_{r} ; s\right)$ and $\eta\left(k_{1}, \ldots, k_{r} ; s\right)$, for general index $\left(k_{1}, \ldots, k_{r}\right)$. These formulas generalize [4, Theorem $\left.9(\mathrm{i})\right]$, and have remarkable similarity in that one obtains the formula for $\eta\left(k_{1}, \ldots, k_{r} ; s\right)$ just by replacing 
multiple zeta values in the one for $\xi\left(k_{1}, \ldots, k_{r} ; s\right)$ with multiple 'zeta-star' values. Recall the multiple zeta-star value is a real number defined by

$$
\zeta^{\star}\left(l_{1}, \ldots, l_{r}\right)=\sum_{1 \leq m_{1} \leq \cdots \leq m_{r}} \frac{1}{m_{1}^{l_{1}} \cdots m_{r}^{l_{r}}}
$$

for $l_{1}, \ldots, l_{r} \in \mathbb{Z}_{\geq 1}$ with $l_{r} \geq 2$. This was first studied (for general $r$ ) by Hoffman in [16].

To state our theorem, we further introduce some notation. For an index set $\mathbf{k}=\left(k_{1}, \ldots, k_{r}\right) \in$ $\mathbb{Z}_{\geq 1}^{r}$, put $\mathbf{k}_{+}=\left(k_{1}, \ldots, k_{r-1}, k_{r}+1\right)$. The usual dual index of an admissible index (i.e. the one that the last entry is greater than one) $\mathbf{k}$ is denoted by $\mathbf{k}^{*}$. For $\mathbf{j}=\left(j_{1}, \ldots, j_{r}\right) \in \mathbb{Z}_{\geq 0}^{r}$, we write $|\mathbf{j}|=j_{1}+\cdots+j_{r}$ and call it the weight of $\mathbf{j}$, and $d(\mathbf{j})=r$, the depth of $\mathbf{j}$. For two such indices $\mathbf{k}$ and $\mathbf{j}$ of the same depth, we denote by $\mathbf{k}+\mathbf{j}$ the index obtained by the component-wise addition, $\mathbf{k}+\mathbf{j}=\left(k_{1}+j_{1}, \ldots, k_{r}+j_{r}\right)$, and by $b(\mathbf{k} ; \mathbf{j})$ the quantity given by

$$
b(\mathbf{k} ; \mathbf{j}):=\prod_{i=1}^{r}\left(\begin{array}{c}
k_{i}+j_{i}-1 \\
j_{i}
\end{array}\right) .
$$

Theorem 2.5. $\quad$ For any index set $\mathbf{k}=\left(k_{1}, \ldots, k_{r}\right) \in \mathbb{Z}_{\geq 1}^{r}$ and any $m \in \mathbb{Z}_{\geq 1}$, we have

$$
\xi\left(k_{1}, \ldots, k_{r} ; m\right)=\sum_{|\mathbf{j}|=m-1, d(\mathbf{j})=n} b\left(\left(\mathbf{k}_{+}\right)^{*} ; \mathbf{j}\right) \zeta\left(\left(\mathbf{k}_{+}\right)^{*}+\mathbf{j}\right)
$$

and

$$
\eta\left(k_{1}, \ldots, k_{r} ; m\right)=(-1)^{r-1} \sum_{|\mathbf{j}|=m-1, d(\mathbf{j})=n} b\left(\left(\mathbf{k}_{+}\right)^{*} ; \mathbf{j}\right) \zeta^{\star}\left(\left(\mathbf{k}_{+}\right)^{*}+\mathbf{j}\right),
$$

where both sums are over all $\mathbf{j} \in \mathbb{Z}_{\geq 0}^{r}$ of weight $m-1$ and depth $n:=d\left(\mathbf{k}_{+}^{*}\right)(=|\mathbf{k}|+1-d(\mathbf{k}))$.

In particular, we have

$$
\xi\left(k_{1}, \ldots, k_{r} ; 1\right)=\zeta\left(\left(\mathbf{k}_{+}\right)^{*}\right) \quad\left(=\zeta\left(\mathbf{k}_{+}\right) \text {, by the duality of multiple zeta values }\right)
$$

and

$$
\eta\left(k_{1}, \ldots, k_{r} ; 1\right)=(-1)^{r-1} \zeta^{\star}\left(\left(\mathbf{k}_{+}\right)^{*}\right) .
$$

In order to prove the theorem, we give certain multiple integral expressions of the functions $\xi\left(k_{1}, \ldots, k_{r} ; s\right)$ and $\eta\left(k_{1}, \ldots, k_{r} ; s\right)$.

Proposition 2.6. Notations being as above, write $\left(\mathbf{k}_{+}\right)^{*}=\left(l_{1}, \ldots, l_{n}\right)$. Then we have, for $\operatorname{Re}(s)>1-r$,

$$
\begin{aligned}
\xi\left(k_{1}, \ldots, k_{r} ; s\right)= & \frac{1}{\prod_{i=1}^{n} \Gamma\left(l_{i}\right) \cdot \Gamma(s)} \int_{0}^{\infty} \cdots \int_{0}^{\infty}\left(x_{1}+\cdots+x_{n}\right)^{s-1} x_{1}^{l_{1}-1} \cdots x_{n}^{l_{n}-1} \\
& \times \frac{1}{e^{x_{1}+\cdots+x_{n}}-1} \cdot \frac{1}{e^{x_{2}+\cdots+x_{n}}-1} \cdots \cdots \frac{1}{e^{x_{n}}-1} d x_{1} \cdots d x_{n} .
\end{aligned}
$$

$$
\begin{aligned}
\eta\left(k_{1}, \ldots, k_{r} ; s\right)= & \frac{(-1)^{r-1}}{\prod_{i=1}^{n} \Gamma\left(l_{i}\right) \cdot \Gamma(s)} \int_{0}^{\infty} \cdots \int_{0}^{\infty}\left(x_{1}+\cdots+x_{n}\right)^{s-1} x_{1}^{l_{1}-1} \cdots x_{n}^{l_{n}-1} \\
& \times \frac{1}{e^{x_{1}+\cdots+x_{n}}-1} \cdot \frac{e^{x_{2}+\cdots+x_{n}}}{e^{x_{2}+\cdots+x_{n}}-1} \cdots \cdots \frac{e^{x_{n}}}{e^{x_{n}}-1} d x_{1} \cdots d x_{n} .
\end{aligned}
$$


Proof. First write the index $\left(k_{1}, \ldots, k_{r}\right)$ as

$$
\left(k_{1}, \ldots, k_{r}\right)=(\underbrace{1, \ldots, 1}_{a_{1}-1}, b_{1}+1, \ldots, \underbrace{1, \ldots, 1}_{a_{h}-1}, b_{h}+1),
$$

with (uniquely determined) integers $h \geq 1, a_{i} \geq 1(1 \leq i \leq h), b_{i} \geq 1(1 \leq i \leq h-1)$, and $b_{h} \geq 0$. Then, by performing the intermediate integrals of repeated $d z /(1-z)$ in the standard iterated integral coming from (2.3), we obtain the following iterated integral expression of the multiple polylogarithm $\operatorname{Li}_{k_{1}, \ldots, k_{r}}(z)$ :

$$
\begin{aligned}
\operatorname{Li}_{k_{1}, \ldots, k_{r}}(z) & =\underbrace{\int_{0}^{z} \frac{d x_{h}}{x_{h}} \int_{0}^{x_{h}} \ldots \int_{0}^{x_{h}} \frac{d x_{h}}{x_{h}} \int_{0}^{x_{h}} \frac{1}{a_{h} !} \log \left(\frac{1-x_{h-1}}{1-x_{h}}\right)^{a_{h}} \frac{d x_{h-1}}{x_{h-1}}}_{b_{h}} \\
& \cdot \underbrace{\int_{0}^{x_{h-1}} \frac{d x_{h-1}}{x_{h-1}} \cdots \cdots \int_{0}^{x_{h-1}} \frac{d x_{h-1}}{x_{h-1}} \int_{0}^{x_{h-1}} \frac{1}{a_{h-1} !} \log \left(\frac{1-x_{h-2}}{1-x_{h-1}}\right)^{a_{h-1}} \frac{d x_{h-2}}{x_{h-2}} \ldots \ldots}_{b_{h-1}-1} \int_{0}^{x_{3}} \frac{d x_{3}}{x_{3}} \int_{0}^{x_{3}} \frac{1}{a_{3} !} \log \left(\frac{1-x_{2}}{1-x_{3}}\right)^{a_{3}} \frac{d x_{2}}{x_{2}} \underbrace{\int_{0}^{x_{2}} \frac{d x_{2}}{x_{2}} \cdots \int_{0}^{x_{2}} \frac{d x_{2}}{x_{2}}}_{b_{3}-1} \\
& \cdots \underbrace{\int_{0}^{x_{3}} \frac{d x_{3}}{x_{3}} \cdots \cdots \int_{0}^{x_{1}} \frac{d x_{1}}{x_{1}} \int_{0}^{x_{1}}}_{b_{2}-1} \frac{(-\log (1-x))^{a_{1}}}{a_{1} !} \frac{d x}{x} .
\end{aligned}
$$

Here, to ease notation, we used the same variable in the repetitions of integrals $\int_{0}^{x} d x / x$, and we understand $x_{h}=z$ if $b_{h}=0$. The paths of integrations are in the domain $\mathbb{C} \backslash[1, \infty)$, and the formula is valid for $z \in \mathbb{C} \backslash[1, \infty)$. We may check this formula by differentiating both sides repeatedly and using (2.3). Putting $z=1-e^{-t}$ and $1-e^{t}$, changing variables accordingly, and suitably labeling the variables, we obtain

$$
\begin{aligned}
& \operatorname{Li}_{k_{1}, \ldots, k_{r}}\left(1-e^{-t}\right)=\int_{0}^{t} \int_{0}^{t_{b_{1}+\cdots+b_{h}}} \cdots \int_{0}^{t_{2}} \underbrace{\frac{1}{e^{t_{b_{1}+\cdots+b_{h}}-1}} \cdots \cdots \frac{1}{e^{t_{b_{1}+\cdots+b_{h-1}+2}}-1}}_{b_{h}-1}
\end{aligned}
$$

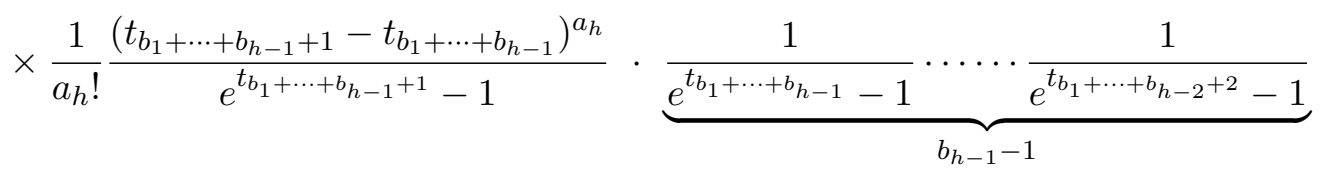

$$
\begin{aligned}
& \times \ldots \ldots \\
& \times \frac{1}{a_{3} !} \frac{\left(t_{b_{1}+b_{2}+1}-t_{b_{1}+b_{2}}\right)^{a_{3}}}{e^{t_{b_{1}+b_{2}+1}}-1} \cdot \underbrace{\frac{1}{e^{t_{b_{1}}+b_{2}}-1} \cdots \cdots \frac{1}{e^{t_{b_{1}+2}}-1}}_{b_{2}-1} \\
& \times \frac{1}{a_{2} !} \frac{\left(t_{b_{1}+1}-t_{b_{1}}\right)^{a_{2}}}{e^{t_{b_{1}+1}}-1} \cdot \underbrace{\frac{1}{e^{t_{b_{1}}}-1} \cdots \cdots \frac{1}{e^{t_{2}}-1}}_{b_{1}-1} \cdot \frac{1}{a_{1} !} \frac{t_{1}^{a_{1}}}{e^{t_{1}}-1} d t_{1} d t_{2} \cdots d t_{b_{1}+\cdots+b_{h}},
\end{aligned}
$$

and

$$
\operatorname{Li}_{k_{1}, \ldots, k_{r}}\left(1-e^{t}\right)=(-1)^{r} \int_{0}^{t} \int_{0}^{t_{b_{1}+\cdots+b_{h}}} \cdots \int_{0}^{t_{2}} \underbrace{\frac{e^{t_{b_{1}}+\cdots+b_{h}}}{e^{t_{b_{1}+\cdots+b_{h}}-1}} \cdots \cdots \frac{e^{t_{b_{1}+\cdots+b_{h-1}+2}}}{e^{t_{b_{1}+\cdots+b_{h-1}+2}}-1}}_{b_{h}-1}
$$




$$
\begin{aligned}
& \times \frac{1}{a_{h} !} \frac{\left(t_{b_{1}+\cdots+b_{h-1}+1}-t_{b_{1}+\cdots+b_{h-1}}\right)^{a_{h}} e^{t_{b_{1}+\cdots+b_{h-1}+1}}}{e^{t_{b_{1}+\cdots+b_{h-1}+1}}-1} \cdot \underbrace{\frac{e^{t_{b_{1}+\cdots+b_{h-1}}}}{e^{t_{b_{1}+\cdots+b_{h-1}}+1}-1} \cdots \frac{e^{t_{b_{1}+\cdots+b_{h-2}+2}}}{e^{t_{b_{1}+\cdots+b_{h-2}+2}+1}}}_{b_{h-1}-1} \\
& \times \ldots \ldots \\
& \times \frac{1}{a_{3} !} \frac{\left(t_{b_{1}+b_{2}+1}-t_{b_{1}+b_{2}}\right)^{a_{3}} e^{t_{b_{1}+b_{2}+1}}}{e^{t_{b_{1}+b_{2}+1}}-1} \cdot \underbrace{\frac{e^{t_{b_{1}+b_{2}}}}{e^{t_{b_{1}+b_{2}}}-1} \cdots \frac{e^{t_{b_{1}+2}}}{e^{t_{b_{1}}+2}-1}}_{b_{2}-1} \\
& \times \frac{1}{a_{2} !} \frac{\left(t_{b_{1}+1}-t_{b_{1}}\right)^{a_{2}} e^{t_{b_{1}+1}}}{e^{t_{b_{1}+1}}-1} \cdot \underbrace{\frac{e^{t_{b_{1}}}}{e^{t_{b_{1}}}-1} \cdots \cdots \frac{e^{t_{2}}}{e^{t_{2}}-1}}_{b_{1}-1} \cdot \frac{1}{a_{1} !} \frac{t_{1}^{a_{1}} e^{t_{1}}}{e^{t_{1}}-1} d t_{1} d t_{2} \cdots d t_{b_{1}+\cdots+b_{h}} .
\end{aligned}
$$

The factor $(-1)^{r}$ on the right of $(2.10)$ comes from $(-1)^{a_{1}+\cdots+a_{h}}=(-1)^{r}$. Plugging $(2.9)$ and (2.10) into the definitions (1.2) and (1.1) respectively and making the change of variables

$t=x_{1}+\cdots+x_{n}, t_{b_{1}+\cdots+b_{h}}=x_{2}+\cdots+x_{n}, t_{b_{1}+\cdots+b_{h}-1}=x_{3}+\cdots+x_{n}, \ldots, t_{2}=x_{n-1}+x_{n}, t_{1}=x_{n}$,

we obtain the proposition. One should note that the dual index $\left(\mathbf{k}_{+}\right)^{*}=\left(l_{1}, \ldots, l_{n}\right)$ is given by

$$
\left(\mathbf{k}_{+}\right)^{*}=(\underbrace{1, \ldots, 1}_{b_{h}}, a_{h}+1, \underbrace{1, \ldots, 1}_{b_{h-1}-1}, a_{h-1}+1, \ldots, \underbrace{1, \ldots, 1}_{b_{1}-1}, a_{1}+1)
$$

and the depth $n$ is equal to $b_{1}+\cdots+b_{h}+1$, and that (the trivial) $x_{i}^{l_{i}-1}=1$ when $l_{i}=1$.

Proof of Theorem 2.5. Set $s=m$ in the integral expressions in the proposition, and expand $\left(x_{1}+\cdots+x_{k}\right)^{m-1}$ by the multinomial theorem. Then the formula in the theorem follows from the lemma below.

Lemma 2.7. For $l_{1}, \ldots, l_{r} \in \mathbb{Z}_{\geq 1}$ with $l_{r} \geq 2$, we have

$$
\zeta\left(l_{1}, \ldots, l_{r}\right)=\frac{1}{\prod_{j=1}^{r} \Gamma\left(l_{j}\right)} \int_{0}^{\infty} \cdots \int_{0}^{\infty} \frac{x_{1}^{l_{1}-1} \cdots x_{r}^{l_{r}-1}}{e^{x_{1}+\cdots+x_{r}}-1} \cdot \frac{1}{e^{x_{2}+\cdots+x_{r}}-1} \cdots \cdots \frac{1}{e^{x_{r}}-1} d x_{1} \cdots d x_{r}
$$

and

$$
\zeta^{\star}\left(l_{1}, \ldots, l_{r}\right)=\frac{1}{\prod_{j=1}^{r} \Gamma\left(l_{j}\right)} \int_{0}^{\infty} \cdots \int_{0}^{\infty} \frac{x_{1}^{l_{1}-1} \cdots x_{r}^{l_{r}-1}}{e^{x_{1}+\cdots+x_{r}}-1} \cdot \frac{e^{x_{2}+\cdots+x_{r}}}{e^{x_{2}+\cdots+x_{r}}-1} \cdots \cdots \frac{e^{x_{r}}}{e^{x_{r}}-1} d x_{1} \cdots d x_{r} .
$$

Proof. The first formula is given in [4, Theorem 3 (i)]. As for the second, we may proceed similarly by using $n^{-s}=\Gamma(s)^{-1} \int_{0}^{\infty} t^{s-1} e^{-n t} d t$ to have

$$
\begin{aligned}
& \zeta^{\star}\left(l_{1}, \ldots, l_{r}\right)=\sum_{m_{1}=1}^{\infty} \sum_{m_{2}, \ldots, m_{r}=0}^{\infty} \frac{1}{m_{1}^{l_{1}}\left(m_{1}+m_{2}\right)^{l_{2}} \cdots \cdots\left(m_{1}+\cdots+m_{r}\right)^{l_{r}}} \\
& =\frac{1}{\prod_{j=1}^{r} \Gamma\left(l_{j}\right)} \sum_{m_{1}=1}^{\infty} \sum_{m_{2}, \ldots, m_{r}=0}^{\infty} \int_{0}^{\infty} \cdots \int_{0}^{\infty} x_{1}^{l_{1}-1} e^{-m_{1} x_{1}} \cdot x_{2}^{l_{2}-1} e^{-\left(m_{1}+m_{2}\right) x_{2}} \ldots \\
& \cdots x_{r}^{l_{r}-1} e^{-\left(m_{1}+\cdots+m_{r}\right) x_{r}} d x_{1} \cdots d x_{r} \\
& =\frac{1}{\prod_{j=1}^{r} \Gamma\left(l_{j}\right)} \sum_{m_{1}=1}^{\infty} \sum_{m_{2}, \ldots, m_{r}=0}^{\infty} \int_{0}^{\infty} \cdots \int_{0}^{\infty} x_{1}^{l_{1}-1} \cdots x_{r}^{l_{r}-1} e^{-m_{1}\left(x_{1}+\cdots+x_{r}\right)} \cdot e^{-m_{2}\left(x_{2}+\cdots+x_{r}\right)} \ldots
\end{aligned}
$$




$$
\begin{aligned}
& \cdots e^{-m_{r} x_{r}} d x_{1} \cdots d x_{r} \\
& =\frac{1}{\prod_{j=1}^{r} \Gamma\left(l_{j}\right)} \int_{0}^{\infty} \cdots \int_{0}^{\infty} \frac{x_{1}^{l_{1}-1} \cdots x_{r}^{l_{r}-1}}{e^{x_{1}+\cdots+x_{r}}-1} \cdot \frac{e^{x_{2}+\cdots+x_{r}}}{e^{x_{2}+\cdots+x_{r}}-1} \cdots \cdots \frac{e^{x_{r}}}{e^{x_{r}}-1} d x_{1} \cdots d x_{r} .
\end{aligned}
$$

We record here one corollary to the theorem in the case of $\eta_{k}(m)$ (compare with the similar formula in $\left[4\right.$, Theorem 9 (i)]). Noting $(k+1)^{*}=(\underbrace{1, \ldots, 1}_{k-1}, 2)$, we have

Corollary 2.8. For $k, m \geq 1$, we have

$$
\eta_{k}(m)=\sum_{\substack{j_{1}, \ldots, j_{k-1} \geq 1, j_{k} \geq 2 \\ j_{1}+\cdots+j_{k}=k+m}}\left(j_{k}-1\right) \zeta^{\star}\left(j_{1}, \ldots, j_{k-1}, j_{k}\right) .
$$

\section{Relations among the functions $\xi, \eta$ and $\zeta$, and their con- sequences to multiple zeta values and multi-poly-Bernoulli numbers}

In this section, we first deduce that each of the functions $\eta$ and $\xi$ can be written as a linear combination of the other by the same formula. This is a consequence of the so-called Landentype connection formula for the multiple polylogarithm $\operatorname{Li}_{k_{1}, \ldots, k_{r}}(z)$. We then establish a formula for $\xi\left(k_{1}, \ldots, k_{r} ; s\right)$ in terms of the single-variable multiple zeta function

$$
\zeta\left(l_{1}, \ldots, l_{r} ; s\right)=\sum_{1 \leq m_{1}<\cdots<m_{r}<m} \frac{1}{m_{1}^{l_{1}} \cdots m_{r}^{l_{r}} m^{s}}
$$

defined for positive integers $l_{1}, \ldots, l_{r}$, the analytic continuation of which has been given in [4] (the analytic continuation of a more general multi-variable multiple zeta function is established in [1]). This answers to the question posed in $\S 5$ of [4]. As a result, the function $\eta\left(k_{1}, \ldots, k_{r} ; s\right)$ can also be written by the multiple zeta functions of the type above. We then give a formula for values at positive integers of $\xi\left(k_{1}, \ldots, k_{r} ; s\right)$, and hence of $\eta\left(k_{1}, \ldots, k_{r} ; s\right)$, in terms of the 'shuffle regularized values' of multiple zeta values, and thereby derive some consequences on the values of $\eta_{k}(s)$.

Let $\mathbf{k}=\left(k_{1}, \ldots, k_{r}\right) \in \mathbb{Z}_{\geq 1}^{r}$ be an index set. Recall that $\mathbf{k}$ is said to be admissible if the last entry $k_{r}$ is greater than 1 , the weight of $\mathbf{k}$ is the sum $k_{1}+\cdots+k_{r}$, and the depth is the length $r$ of the index. For two indices $\mathbf{k}$ and $\mathbf{k}^{\prime}$ of the same weight, we say $\mathbf{k}^{\prime}$ refines $\mathbf{k}$, denoted $\mathbf{k} \preceq \mathbf{k}^{\prime}$, if $\mathbf{k}$ is obtained from $\mathbf{k}^{\prime}$ by replacing some commas by +'s. For example, $(5)=(2+3) \preceq(2,3),(2,3)=(1+1,2+1) \preceq(1,1,2,1)$, etc. The standard expression of a multiple zeta-star value as a sum of multiple zeta values is written as

$$
\zeta^{\star}(\mathbf{k})=\sum_{\substack{\mathbf{k}^{\prime} \leq \mathbf{k} \\ \text { admissible }}} \zeta\left(\mathbf{k}^{\prime}\right),
$$

where the sum on the right runs over the admissible indices $\mathbf{k}^{\prime}$ such that $\mathbf{k}$ refines $\mathbf{k}^{\prime}$.

The following formula is known as the Landen connection formula for the multiple polylogarithm ([26, Proposition 9]). 
Lemma 3.1. For any index $\mathbf{k}$ of depth $r$, we have

$$
\mathrm{Li}_{\mathbf{k}}\left(\frac{z}{z-1}\right)=(-1)^{r} \sum_{\mathbf{k} \preceq \mathbf{k}^{\prime}} \operatorname{Li}_{\mathbf{k}^{\prime}}(z) .
$$

We can prove this by induction on weight and by using (2.3), see [26].

By using this and noting $z /(z-1)=1-e^{t}\left(\right.$ resp. $\left.1-e^{-t}\right)$ if $z=1-e^{-t}\left(\right.$ resp. $\left.1-e^{t}\right)$, we immediately obtain the following proposition.

Proposition 3.2. Let $\mathbf{k}$ be any index set and $r$ its depth. We have the relations

$$
\eta(\mathbf{k} ; s)=(-1)^{r-1} \sum_{\mathbf{k} \preceq \mathbf{k}^{\prime}} \xi\left(\mathbf{k}^{\prime} ; s\right)
$$

and

$$
\xi(\mathbf{k} ; s)=(-1)^{r-1} \sum_{\mathbf{k} \preceq \mathbf{k}^{\prime}} \eta\left(\mathbf{k}^{\prime} ; s\right) .
$$

Corollary 3.3. Let $k$ be a positive integer. Then we have

$$
\eta_{k}(s)=\sum_{\mathbf{k}: \text { weight } k} \xi(\mathbf{k} ; s)
$$

and

$$
\xi_{k}(s)=\sum_{\mathbf{k}: \text { weight } k} \eta(\mathbf{k} ; s),
$$

where the sums run over all indices of weight $k$. Here we have written $\xi_{k}(s)$ for $\xi(k ; s)$.

Proof. The index $(k)$ is of depth 1 and all indices of weight $k$ (admissible or non-admissible) refine $(k)$.

We mention here that, also by taking $\mathbf{k}=(k)$ in Lemma 3.1 and setting $z=1-e^{t}$ or $1-e^{-t}$, one immediately obtains a kind of sum formulas for multi-poly-Bernoulli numbers as follows (compare with similar formulas in [17, Theorem 3.1]).

Corollary 3.4. For $k \geq 1$ and $m \geq 0$, we have

$$
B_{m}^{(k)}=(-1)^{m} \sum_{\substack{k_{1}+\cdots+k_{r}=k \\ k_{i}, r \geq 1}} C_{m}^{\left(k_{1}, \ldots, k_{r}\right)}
$$

and

$$
C_{m}^{(k)}=(-1)^{m} \sum_{\substack{k_{1}+\cdots, k_{r}=k \\ k_{i}, r \geq 1}} B_{m}^{\left(k_{1}, \ldots, k_{r}\right)} .
$$

Next, we prove an Euler-type connection formula for the multiple polylogarithm. If an index $\mathbf{k}$ is of weight $|\mathbf{k}|$, we also say the multiple zeta value $\zeta(\mathbf{k})$ is of weight $|\mathbf{k}|$. 
Lemma 3.5. Let $\mathbf{k}$ be any index. Then we have

$$
\mathrm{Li}_{\mathbf{k}}(1-z)=\sum_{\mathbf{k}^{\prime}, j \geq 0} c_{\mathbf{k}}\left(\mathbf{k}^{\prime} ; j\right) \operatorname{Li}_{\underbrace{1, \ldots, 1}_{j}}(1-z) \operatorname{Li}_{\mathbf{k}^{\prime}}(z),
$$

where the sum on the right runs over indices $\mathbf{k}^{\prime}$ and integers $j \geq 0$ that satisfy $\left|\mathbf{k}^{\prime}\right|+j \leq|\mathbf{k}|$, and $c_{\mathbf{k}}\left(\mathbf{k}^{\prime} ; j\right)$ is a $\mathbb{Q}$-linear combination of multiple zeta values of weight $|\mathbf{k}|-\left|\mathbf{k}^{\prime}\right|-j$. We understand $\operatorname{Li}_{\emptyset}(z)=1$ and $|\emptyset|=0$ for the empty index $\emptyset$, and the constant 1 is regarded as a multiple zeta value of weight 0 .

Proof. We proceed by induction on the weight of $\mathbf{k}$. When $\mathbf{k}=(1)$, the trivial identity $\operatorname{Li}_{1}(1-$ $z)=\operatorname{Li}_{1}(1-z)$ is the one asserted. Suppose the weight $|\mathbf{k}|$ of $\mathbf{k}$ is greater than 1 and assume the statement holds for any index of weight less than $|\mathbf{k}|$. For $\mathbf{k}=\left(k_{1}, \ldots, k_{r}\right)$, set $\mathbf{k}_{-}=$ $\left(k_{1}, \ldots, k_{r-1}, k_{r}-1\right)$ and $\mathbf{k}_{+}=\left(k_{1}, \ldots, k_{r-1}, k_{r}+1\right)$.

First assume that $\mathbf{k}$ is admissible. Then, by (2.3) and induction hypothesis, we have

$$
\frac{d}{d z} \operatorname{Li}_{\mathbf{k}}(1-z)=-\frac{\operatorname{Li}_{\mathbf{k}_{-}}(1-z)}{1-z}=-\frac{1}{1-z} \sum_{\mathbf{l}, j \geq 0} c_{\mathbf{k}_{-}}(\mathbf{l} ; j) \operatorname{Li}_{j}^{i_{1, \ldots, 1}}(1-z) \operatorname{Li}_{\mathbf{l}}(z),
$$

the right-hand side being of a desired form. Here, again by (2.3), we see that

$$
\frac{1}{1-z} \operatorname{Li}_{j} \underbrace{}_{j, \ldots, 1}(1-z) \operatorname{Li}_{\mathbf{l}}(z)=\frac{d}{d z}\left(\sum_{i=0}^{j} \operatorname{Li}_{\underbrace{1, \ldots, 1}_{j-i}}(1-z) \operatorname{Li}_{\mathbf{l}, 1+i}(z)\right) .
$$

We therefore conclude

$$
\operatorname{Li}_{\mathbf{k}}(1-z)=-\sum_{\mathbf{l}, j \geq 0} c_{\mathbf{k}_{-}}(\mathbf{l} ; j) \sum_{i=0}^{j} \operatorname{Li}_{\underbrace{1, \ldots, 1}_{j-i}}(1-z) \operatorname{Li}_{\mathbf{l}, 1+i}(z)+C
$$

with some constant $C$. Since $\lim _{z \rightarrow 0} \operatorname{Li}_{\underbrace{1, \ldots, 1}_{j-i}}(1-z) \operatorname{Li}_{1,1+i}(z)=0$, we find $C=\zeta(\mathbf{k})$ by setting $z \rightarrow 0$, and obtain the desired expression for $\operatorname{Li}_{\mathbf{k}}(1-z)$.

When $\mathbf{k}$ is not necessarily admissible, write $\mathbf{k}=(\mathbf{k}_{0}, \underbrace{1, \ldots, 1}_{q})$ with an admissible $\mathbf{k}_{0}$ and $q \geq 0$. We prove the identity by induction on $q$. The case $q=0$ ( $\mathbf{k}$ is admissible) is already done. Suppose $q \geq 1$ and assume the claim is true for smaller $q$. Then by assumption we have the expression

$$
\operatorname{Li}_{\mathbf{k}_{0}, 1, \ldots, 1}(1-z)=\sum_{q-1} c_{\mathbf{k}^{\prime}}(\mathbf{m} ; j) \operatorname{Li}_{\underbrace{1, \ldots, 1}_{j}}^{1_{1, \ldots}}(1-z) \operatorname{Li}_{\mathbf{m}}(z),
$$

where we have put $\mathbf{k}^{\prime}=(\mathbf{k}_{0}, \underbrace{1, \ldots, 1}_{q-1})$. We multiply $\operatorname{Li}_{1}(1-z)$ on both sides. Then, by the shuffle product, the left-hand side becomes the sum of the form

$$
q \operatorname{Li}_{\mathbf{k}}(1-z)+\sum_{\mathbf{k}_{0}^{\prime} \text { :admissible }} \operatorname{Li}_{\mathbf{k}_{0}^{\prime}, \underbrace{1, \ldots, 1}_{q-1}}(1-z),
$$

and each term in the sum is written in the claimed form by induction hypothesis. On the other hand, the right-hand side becomes also of the form desired because

$$
\operatorname{Li}_{1}(1-z) \operatorname{Li}_{\underbrace{1, \ldots, 1}_{j}}^{\underbrace{}_{1,1}}(1-z)=(j+1) \operatorname{Li}_{\underbrace{1, \ldots, 1}_{j+1}}(1-z) .
$$

Hence $\operatorname{Li}_{\mathbf{k}}(1-z)$ is of the form as claimed. 
With the lemma, we are now able to establish the following (see $[4, \S 5$, Problem (i)]).

Theorem 3.6. Let $\mathbf{k}$ be any index set. The function $\xi(\mathbf{k} ; s)$ can be written in terms of multiple zeta functions as

$$
\xi(\mathbf{k} ; s)=\sum_{\mathbf{k}^{\prime}, j \geq 0} c_{\mathbf{k}}\left(\mathbf{k}^{\prime} ; j\right)\left(\begin{array}{c}
s+j-1 \\
j
\end{array}\right) \zeta\left(\mathbf{k}^{\prime} ; s+j\right) .
$$

Here, the sum is over indices $\mathbf{k}^{\prime}$ and integers $j \geq 0$ satisfying $\left|\mathbf{k}^{\prime}\right|+j \leq|\mathbf{k}|$, and $c_{\mathbf{k}}\left(\mathbf{k}^{\prime} ; j\right)$ is a $\mathbb{Q}$-linear combination of multiple zeta values of weight $|\mathbf{k}|-\left|\mathbf{k}^{\prime}\right|-j$. The index $\mathbf{k}^{\prime}$ may be $\emptyset$ and for this we set $\zeta(\emptyset ; s+j)=\zeta(s+j)$.

Proof. By setting $z=e^{-t}$ in the lemma and using

$$
\operatorname{Li}_{\underbrace{1, \ldots, 1}_{j}}(z)=\frac{(-\log (1-z))^{j}}{j !},
$$

we have

$$
\mathrm{Li}_{\mathbf{k}}\left(1-e^{-t}\right)=\sum_{\mathbf{k}^{\prime}, j \geq 0} c_{\mathbf{k}}\left(\mathbf{k}^{\prime} ; j\right) \frac{t^{j}}{j !} \mathrm{Li}_{\mathbf{k}^{\prime}}\left(e^{-t}\right) .
$$

Substituting this into the definition (1.2) of $\xi(\mathbf{k} ; s)$ and using the formula ([4, Proposition 2, (i)])

$$
\zeta(\mathbf{k} ; s)=\frac{1}{\Gamma(s)} \int_{0}^{\infty} \frac{t^{s-1}}{e^{t}-1} \mathrm{Li}_{\mathbf{k}}\left(e^{-t}\right) d t,
$$

we immediately obtain the theorem.

Remark 3.7. This theorem generalizes [4, Theorem 8], where the corresponding formula for $\mathrm{Li}_{\underbrace{1, \ldots, 1}_{1-1}, k}(1-z)$ is

$$
\begin{aligned}
\operatorname{Li}_{\underbrace{}_{r-1}, \ldots, 1, k}(1-z)= & (-1)^{k-1} \sum_{\substack{j_{1}+\cdots+j_{k}=r+k \\
\forall j_{i} \geq 1}} \operatorname{Li}_{j_{j_{k}-1}, \ldots, 1}(1-z) \operatorname{Li}_{j_{1}, \ldots, j_{k-1}}(z) \\
& +\sum_{j=0}^{k-2}(-1)^{j} \zeta(\underbrace{1, \ldots, 1}_{r-1}, k-j) \underbrace{1, \ldots, 1}_{j}(z) .
\end{aligned}
$$

As pointed out by Shu Oi, one can deduce Lemma 3.5 by induction using [27, Prop. 5]. However, to describe the right-hand side of the lemma explicitly is a different problem and neither proof gives such a formula in general. See also [25] for a related topic.

Example 3.8. Apart from the trivial case $(1, \ldots, 1)$, examples of the identity in Lemma 3.5 up to weight 4 are:

$$
\begin{aligned}
\mathrm{Li}_{2}(1-z)= & -\mathrm{Li}_{2}(z)-\mathrm{Li}_{1}(1-z) \operatorname{Li}_{1}(z)+\zeta(2) \\
\mathrm{Li}_{3}(1-z)= & \mathrm{Li}_{1,2}(z)+\mathrm{Li}_{2,1}(z)+\mathrm{Li}_{1}(1-z) \mathrm{Li}_{1,1}(z)-\zeta(2) \mathrm{Li}_{1}(z)+\zeta(3), \\
\mathrm{Li}_{1,2}(1-z)= & -\mathrm{Li}_{3}(z)-\mathrm{Li}_{1}(1-z) \operatorname{Li}_{2}(z)-\mathrm{Li}_{1,1}(1-z) \mathrm{Li}_{1}(z)+\zeta(3), \\
\mathrm{Li}_{2,1}(1-z)= & 2 \mathrm{Li}_{3}(z)+\mathrm{Li}_{1}(1-z) \mathrm{Li}_{2}(z)+\zeta(2) \operatorname{Li}_{1}(1-z)-2 \zeta(3) \\
\mathrm{Li}_{4}(1-z)= & -\mathrm{Li}_{1,1,2}(z)-\mathrm{Li}_{1,2,1}(z)-\mathrm{Li}_{2,1,1}(z)-\mathrm{Li}_{1}(1-z) \mathrm{Li}_{1,1,1}(z) \\
& +\zeta(2) \mathrm{Li}_{1,1}(z)-\zeta(3) \mathrm{Li}_{1}(z)+\zeta(4),
\end{aligned}
$$




$$
\begin{aligned}
& \operatorname{Li}_{1,3}(1-z)=\operatorname{Li}_{1,3}(z)+\operatorname{Li}_{2,2}(z)+\operatorname{Li}_{3,1}(z)+\operatorname{Li}_{1}(1-z) \operatorname{Li}_{1,2}(z)+\operatorname{Li}_{1}(1-z) \operatorname{Li}_{2,1}(z) \\
& +\mathrm{Li}_{1,1}(1-z) \mathrm{Li}_{1,1}(z)-\zeta(3) \mathrm{Li}_{1}(z)+\frac{1}{4} \zeta(4) \\
& \operatorname{Li}_{2,2}(1-z)=-\mathrm{Li}_{2,2}(z)-2 \operatorname{Li}_{3,1}(z)-\operatorname{Li}_{1}(1-z) \operatorname{Li}_{2,1}(z)-\zeta(2) \operatorname{Li}_{1}(1-z) \operatorname{Li}_{1}(z) \\
& -\zeta(2) \operatorname{Li}_{2}(z)+2 \zeta(3) \operatorname{Li}_{1}(z)+\frac{3}{4} \zeta(4) \\
& \mathrm{Li}_{3,1}(1-z)=-2 \mathrm{Li}_{1,3}(z)-\mathrm{Li}_{2,2}(z)-\mathrm{Li}_{1}(1-z) \mathrm{Li}_{1,2}(z)+\zeta(2) \mathrm{Li}_{2}(z) \\
& +\zeta(3) \operatorname{Li}_{1}(1-z)-\frac{5}{4} \zeta(4) \\
& \operatorname{Li}_{1,1,2}(1-z)=-\mathrm{Li}_{4}(z)-\operatorname{Li}_{1}(1-z) \operatorname{Li}_{3}(z)-\mathrm{Li}_{1,1}(1-z) \operatorname{Li}_{2}(z) \\
& -\mathrm{Li}_{1,1,1}(1-z) \mathrm{Li}_{1}(z)+\zeta(4) \\
& \operatorname{Li}_{1,2,1}(1-z)=3 \mathrm{Li}_{4}(z)+2 \mathrm{Li}_{1}(1-z) \operatorname{Li}_{3}(z)+\mathrm{Li}_{1,1}(1-z) \operatorname{Li}_{2}(z)+\zeta(3) \operatorname{Li}_{1}(1-z) \\
& -3 \zeta(4) \text {, } \\
& \operatorname{Li}_{2,1,1}(1-z)=-3 \operatorname{Li}_{4}(z)-\operatorname{Li}_{1}(1-z) \operatorname{Li}_{3}(z)+\zeta(2) \operatorname{Li}_{1,1}(1-z)-2 \zeta(3) \operatorname{Li}_{1}(1-z) \\
& +3 \zeta(4) \text {. }
\end{aligned}
$$

Accordingly, we have

$$
\begin{aligned}
& \xi(2 ; s)=-\zeta(2 ; s)-s \zeta(1 ; s+1)+\zeta(2) \zeta(s), \\
& \xi(3 ; s)=\zeta(1,2 ; s)+\zeta(2,1 ; s)+s \zeta(1,1 ; s+1)-\zeta(2) \zeta(1 ; s)+\zeta(3) \zeta(s), \\
& \xi(1,2 ; s)=-\zeta(3 ; s)-s \zeta(2 ; s+1)-\frac{s(s+1)}{2} \zeta(1 ; s+2)+\zeta(3) \zeta(s), \\
& \xi(2,1 ; s)=2 \zeta(3 ; s)+s \zeta(2 ; s+1)+\zeta(2) s \zeta(s+1)-2 \zeta(3) \zeta(s), \\
& \xi(4 ; s)=-\zeta(1,1,2 ; s)-\zeta(1,2,1 ; s)-\zeta(2,1,1 ; s)-s \zeta(1,1,1 ; s+1) \\
& +\zeta(2) \zeta(1,1 ; s)-\zeta(3) \zeta(1 ; s)+\zeta(4) \zeta(s), \\
& \xi(1,3 ; s)=\zeta(1,3 ; s)+\zeta(2,2 ; s)+\zeta(3,1 ; s)+s \zeta(1,2 ; s+1)+s \zeta(2,1 ; s+1) \\
& +\frac{s(s+1)}{2} \zeta(1,1 ; s+2)-\zeta(3) \zeta(1 ; s)+\frac{1}{4} \zeta(4) \zeta(s), \\
& \xi(2,2 ; s)=-\zeta(2,2 ; s)-2 \zeta(3,1 ; s)-s \zeta(2,1 ; s+1)-\zeta(2) s \zeta(1 ; s+1) \\
& -\zeta(2) \zeta(2 ; s)+2 \zeta(3) \zeta(1 ; s)+\frac{3}{4} \zeta(4) \zeta(s), \\
& \xi(3,1 ; s)=-2 \zeta(1,3 ; s)-\zeta(2,2 ; s)-s \zeta(1,2 ; s+1)+\zeta(2) \zeta(2 ; s) \\
& +\zeta(3) s \zeta(s+1)-\frac{5}{4} \zeta(4) \zeta(s) \\
& \xi(1,1,2 ; s)=-\zeta(4 ; s)-s \zeta(3 ; s+1)-\frac{s(s+1)}{2} \zeta(2 ; s+2) \\
& -\frac{s(s+1)(s+2)}{6} \zeta(1 ; s+3)+\zeta(4) \zeta(s), \\
& \xi(1,2,1 ; s)=3 \zeta(4 ; s)+2 s \zeta(3 ; s+1)+\frac{s(s+1)}{2} \zeta(2 ; s+2)+\zeta(3) s \zeta(s+1) \\
& -3 \zeta(4) \zeta(s) \\
& \xi(2,1,1 ; s)=-3 \zeta(4 ; s)-s \zeta(3 ; s+1)+\zeta(2) \frac{s(s+1)}{2} \zeta(s+2)-2 \zeta(3) s \zeta(s+1) \\
& +3 \zeta(4) \zeta(s) \text {. }
\end{aligned}
$$

From these and (3.5) of Corollary 3.3, we have for instance

$$
\eta_{2}(s)=\xi(2 ; s)+\xi(1,1 ; s)
$$




$$
\begin{aligned}
= & -\zeta(2 ; s)-s \zeta(1 ; s+1)+\zeta(2) \zeta(s)+\frac{s(s+1)}{2} \zeta(s+2), \\
\eta_{3}(s)= & \xi(3 ; s)+\xi(1,2 ; s)+\xi(2,1 ; s)+\xi(1,1,1 ; s) \\
= & \zeta(3 ; s)+\zeta(1,2 ; s)+\zeta(2,1 ; s)+s \zeta(1,1 ; s+1)-\frac{s(s+1)}{2} \zeta(1 ; s+2) \\
& \quad-\zeta(2) \zeta(1 ; s)+\zeta(2) s \zeta(s+1)+\frac{s(s+1)(s+2)}{6} \zeta(s+3), \\
\eta_{4}(s)= & \xi(4 ; s)+\xi(1,3 ; s)+\xi(2,2 ; s)+\xi(3,1 ; s)+\xi(1,1,2 ; s)+\xi(1,2,1 ; s) \\
& \quad+\xi(2,1,1 ; s)+\xi(1,1,1,1 ; s) \\
= & -\zeta(4 ; s)-\zeta(1,3 ; s)-\zeta(2,2 ; s)-\zeta(3,1 ; s)-\zeta(1,1,2 ; s)-\zeta(1,2,1 ; s) \\
& \quad-\zeta(2,1,1 ; s)-s \zeta(1,1,1 ; s+1)+\zeta(2) \zeta(1,1 ; s)+\frac{s(s+1)}{2} \zeta(1,1 ; s+2) \\
& \quad-\zeta(2) s \zeta(1 ; s+1)+\zeta(2) \frac{s(s+1)}{2} \zeta(s+2)-\frac{s(s+1)(s+2)}{6} \zeta(1 ; s+3) \\
& +\frac{7}{4} \zeta(4) \zeta(s)+\frac{s(s+1)(s+2)(s+3)}{24} \zeta(s+4) .
\end{aligned}
$$

Before closing this section, we present a curious observation. Recall the formula

$$
\xi_{k}(m)=\zeta^{\star}(\underbrace{1, \ldots, 1}_{m-1}, k+1)
$$

discovered by Ohno [24]. Comparing this with the two formulas (2.11) and [4, Corollary 10], one may expect

$$
\eta_{k}(m) \stackrel{?}{=} \zeta(\underbrace{1, \ldots, 1}_{m-1}, k+1) .
$$

This is not true in fact. However, we found experimentally the identities

$$
\eta_{k}(m)=\eta_{m}(k)
$$

and

$$
\sum_{j=1}^{k-1}(-1)^{j-1} \eta_{k-j}(j)= \begin{cases}2\left(1-2^{1-k}\right) \zeta(k) & (k: \text { even }) \\ 0 & (k: \text { odd }) .\end{cases}
$$

These are respectively analogous to the duality relation

$$
\zeta(\underbrace{1, \ldots, 1}_{m-1}, k+1)=\zeta(\underbrace{1, \ldots, 1}_{k-1}, m+1)
$$

and the relation

$$
\sum_{j=1}^{k-1}(-1)^{j-1} \zeta(\underbrace{1, \ldots, 1}_{j-1}, k-j+1)= \begin{cases}2\left(1-2^{1-k}\right) \zeta(k) & (k: \text { even }), \\ 0 & (k: \text { odd }),\end{cases}
$$

which is a special case of the Le-Murakami relation [23] (or one can derive this from the wellknown generating series identity [2], [11]

$$
1-\sum_{k>j \geq 1} \zeta(\underbrace{1, \ldots, 1}_{j-1}, k-j+1) X^{k-j} Y^{j}=\frac{\Gamma(1-X) \Gamma(1-Y)}{\Gamma(1-X-Y)}
$$

by setting $Y=-X$ and using the reflection formula for the gamma function.)

We are still not able to prove $(3.11)^{*}$, but could prove (3.12) by using the following general

*Quite recently, Shuji Yamamoto communicated to the authors that he found a proof. 
formula for the value $\xi(\mathbf{k} ; m)$ and the relation (3.3) in Proposition 3.2. We shall discuss this and other aspects of 'height one' multiple zeta values in more detail in a subsequent paper [22].

Proposition 3.9. Let $\mathbf{k}$ be any index and $m \geq 1$ an integer. Then we have

$$
\xi(\mathbf{k} ; m)=(-1)^{m-1} \zeta^{\amalg}(\mathbf{k}_{+}, \underbrace{1, \ldots, 1}_{m-1}),
$$

where $\zeta^{\mathrm{II}}$ stands for the 'shuffle regularized' value, which is the constant term of the shuffle regularized polynomial defined in [19].

Proof. By making the change of variable $x=1-e^{-t}$ in the definition (1.2), we have

$$
\xi(\mathbf{k} ; s)=\frac{1}{\Gamma(s)} \int_{0}^{1}(-\log (1-x))^{s-1} \operatorname{Li}_{\mathbf{k}}(x) \frac{d x}{x} .
$$

Put $s=m$ and use (3.10) to obtain

$$
\xi(\mathbf{k} ; m)=\int_{0}^{1} \operatorname{Li}_{m-1}^{\underbrace{}_{1, \ldots, 1}}(x) \operatorname{Li}_{\mathbf{k}}(x) \frac{d x}{x} .
$$

The regularization formula [19, Eq. (5.2)], together with the shuffle product of $\operatorname{Li}_{i_{m-1}^{1, \ldots, 1}}(x) \operatorname{Li}_{\mathbf{k}}(x)$, immediately gives (3.13).

By using (3.13) and (3.5), we can write $\eta_{k}(m)$ in terms of shuffle regularized values. The following expression seems to follow from that formula by taking the dual, but we have not yet worked it out in detail.

$$
\eta_{k}(m) \stackrel{?}{=}\left(\begin{array}{c}
m+k \\
k
\end{array}\right) \zeta(m+k)-\sum_{\substack{2 \leq r \leq k+1 \\
j_{1}+\cdots+j_{r}=m+k-r-1}}\left(\begin{array}{c}
j_{1}+\cdots+j_{r-1} \\
k-r+1
\end{array}\right) \zeta\left(j_{1}+1, \cdots, j_{r-1}+1, j_{r}+2\right) .
$$

\section{The function $\eta\left(k_{1}, \ldots, k_{r} ; s\right)$ for non-positive indices}

In this section, as in the case of positive indices, we construct $\eta$-functions with non-positive indices. It is known that $\mathrm{Li}_{-k}(z)$ can be expressed as

$$
\mathrm{Li}_{-k}(z)=\frac{P(z ; k)}{(1-z)^{k}}
$$

for $k \in \mathbb{Z}_{\geq 0}$, where $P(x ; k) \in \mathbb{Z}[x]$ is a monic polynomial satisfying

$$
\begin{aligned}
& \operatorname{deg} P(x ; k)= \begin{cases}1 & (k=0) \\
k & (k \geq 1),\end{cases} \\
& x \mid P(x ; k)
\end{aligned}
$$

(see, for example, Shimura [29, Equations $(2.17),(4.2)$ and $(4.6)]$; Note that the above $P(x ; k)$ coincides with $x P_{k+1}(x)$ in [29]). We first extend this fact to multiple polylogarithms with non-positive indices as follows. 
Lemma 4.1. For $k_{1}, \ldots, k_{r} \in \mathbb{Z}_{\geq 0}$, there exists a polynomial $P\left(x ; k_{1}, \ldots, k_{r}\right) \in \mathbb{Z}[x]$ such that

$$
\begin{aligned}
& \mathrm{Li}_{-k_{1}, \ldots,-k_{r}}(z)=\frac{P\left(z ; k_{1}, \ldots, k_{r}\right)}{(1-z)^{k_{1}+\cdots+k_{r}+r}}, \\
& \operatorname{deg} P\left(x ; k_{1}, \ldots, k_{r}\right)= \begin{cases}r & \left(k_{1}=\cdots=k_{r}=0\right) \\
k_{1}+\cdots+k_{r}+r-1 & (\text { otherwise }),\end{cases} \\
& x^{r} \mid P\left(x ; k_{1}, \ldots, k_{r}\right) .
\end{aligned}
$$

More explicitly, $P(x ; \underbrace{0,0, \ldots, 0}_{r})=x^{r}$.

Proof. We prove this lemma by the double induction on $r \geq 1$ and $K=k_{1}+\cdots+k_{r} \geq 0$. The case $r=1$ is as mentioned above. For $r \geq 2$, we assume the case of $r-1$ holds and consider the case of $r$. When $K=k_{1}+\cdots+k_{r}=0$, namely $k_{1}=\cdots=k_{r}=0$, we have

$$
\begin{aligned}
\operatorname{Li}_{0, \ldots, 0}(z) & =\sum_{m_{1}<\cdots<m_{r}} x^{m_{r}}=\sum_{m_{1}<\cdots<m_{r-1}} \sum_{m_{r}=m_{r-1}+1}^{\infty} z^{m_{r}} \\
& =\frac{z}{1-z} \sum_{m_{1}<\cdots<m_{r-1}} z^{m_{r-1}}=\cdots=\frac{z^{r}}{(1-z)^{r}},
\end{aligned}
$$

which implies (4.1)-(4.3) hold, and also $P(x ; 0, \ldots, 0)=x^{r}$. Hence we assume the case $K=$ $k_{1}+\cdots+k_{r}-1$ holds and consider the case $K=k_{1}+\cdots+k_{r}(\geq 1)$. We consider the two cases $k_{r}=0$ and $k_{r} \geq 1$ separately. First we assume $k_{r}=0$. Then, by induction hypothesis, we have

$$
\begin{aligned}
\mathrm{Li}_{-k_{1}, \ldots,-k_{r-1}, 0}(z) & =\sum_{m_{1}<\cdots<m_{r-1}} m_{1}^{k_{1}} \cdots m_{r-1}^{k_{r-1}} \sum_{m_{r}=m_{r-1}+1}^{\infty} z^{m_{r}} \\
& =\frac{z}{1-z} \sum_{m_{1}<\cdots<m_{r-1}} m_{1}^{k_{1}} \cdots m_{r-1}^{k_{r-1}} z^{m_{r-1}} \\
& =\frac{z}{1-z} \frac{P\left(z: k_{1}, \ldots, k_{r-1}\right)}{(1-z)^{k_{1}+\cdots+k_{r-1}+r-1}} .
\end{aligned}
$$

Let $P\left(z ; k_{1}, \ldots, k_{r-1}, 0\right)=z P\left(z ; k_{1}, \ldots, k_{r-1}\right)$. Then (4.1)-(4.3) hold.

Next we assume $k_{r} \geq 1$. Then, using the same formula as in (2.3) and the induction hypothesis, we have

$$
\begin{aligned}
& \mathrm{Li}_{-k_{1}, \ldots,-k_{r-1},-k_{r}}(z)=z \frac{d}{d z} \mathrm{Li}_{-k_{1}, \ldots,-k_{r}+1}(z) \\
& =z \frac{d}{d z}\left(\frac{P\left(z: k_{1}, \ldots, k_{r}-1\right)}{(1-z)^{k_{1}+\cdots+k_{r}-1+r}}\right) \\
& =\frac{z\left\{P^{\prime}\left(z: k_{1}, \ldots, k_{r}-1\right)(1-z)+\left(k_{1}+\cdots+k_{r}-1+r\right) P\left(z ; k_{1}, \ldots, k_{r}-1\right)\right\}}{(1-z)^{k_{1}+\cdots+k_{r}+r}} .
\end{aligned}
$$

If $k_{1}=\cdots=k_{r-1}=0$ and $k_{r}=1$, then the numerator, that is, $P(0, \ldots, 0,-1)$ equals $r z^{r}$, using the above results. If not, the degree of the numerator equals $k_{1}+\cdots+k_{r}+r-1$ by induction hypothesis. The both cases satisfy (4.1)-(4.3). This completes the proof of the lemma.

Remark 4.2. In the case $r \geq 2, P\left(x ; k_{1}, \ldots, k_{r}\right)$ is not necessarily a monic polynomial. For example, we have $\operatorname{Li}_{0,-1}(z)=2 z^{2} /(1-z)^{3}$, so $P(x ; 0,1)=2 x^{2}$. 
We obtain from (4.1) and (4.2) that

$$
\mathrm{Li}_{-k_{1}, \ldots,-k_{r}}\left(1-e^{t}\right)=\frac{P\left(1-e^{t} ; k_{1}, \ldots, k_{r}\right)}{e^{\left(k_{1}+\cdots+k_{r}+r\right) t}}= \begin{cases}O(1) & \left(k_{1}=\cdots=k_{r}=0\right) \\ O\left(e^{-t}\right) & (\text { otherwise })\end{cases}
$$

as $t \rightarrow \infty$, and from (4.3) that

$$
\mathrm{Li}_{-k_{1}, \ldots,-k_{r}}\left(1-e^{t}\right)=O\left(t^{r}\right) \quad(t \rightarrow 0) .
$$

Therefore we can define the following.

Definition 4.3. For $k_{1}, \ldots, k_{r} \in \mathbb{Z}_{\geq 0}$, define

$$
\eta\left(-k_{1}, \ldots,-k_{r} ; s\right)=\frac{1}{\Gamma(s)} \int_{0}^{\infty} t^{s-1} \frac{\mathrm{Li}_{-k_{1}, \ldots,-k_{r}}\left(1-e^{t}\right)}{1-e^{t}} d t
$$

for $s \in \mathbb{C}$ with $\operatorname{Re}(s)>1-r$. In the case $r=1$, denote $\eta(-k ; s)$ by $\eta_{-k}(s)$.

We see that the integral on the right-hand side of (4.6) is absolutely convergent for $\operatorname{Re}(s)>$ $1-r$. Hence $\eta\left(-k_{1}, \ldots,-k_{r} ; s\right)$ is holomorphic for $\operatorname{Re}(s)>1-r$. By the same method as in the proof of Theorem 2.3 for $\eta\left(k_{1}, \ldots, k_{r} ; s\right)$, we can similarly obtain the following.

Theorem 4.4. For $k_{1}, \ldots, k_{r} \in \mathbb{Z}_{\geq 0}, \eta\left(-k_{1}, \ldots,-k_{r} ; s\right)$ can be analytically continued to an entire function on the whole complex plane, and satisfies

$$
\eta\left(-k_{1}, \ldots,-k_{r} ;-m\right)=B_{m}^{\left(-k_{1}, \ldots,-k_{r}\right)} \quad\left(m \in \mathbb{Z}_{\geq 0}\right) .
$$

In particular, $\eta_{-k}(-m)=B_{m}^{(-k)}\left(k \in \mathbb{Z}_{\geq 0}, m \in \mathbb{Z}_{\geq 0}\right)$.

It should be noted that $\xi\left(-k_{1}, \ldots,-k_{r} ; s\right)$ cannot be defined by replacing $\left\{k_{j}\right\}$ by $\left\{-k_{j}\right\}$ in (1.2). In fact, even if $r=1$ and $k=0$ in (1.2), we see that

$$
\xi_{0}(s)=\frac{1}{\Gamma(s)} \int_{0}^{\infty} t^{s-1} \frac{\operatorname{Li}_{0}\left(1-e^{-t}\right)}{e^{t}-1} d t=\frac{1}{\Gamma(s)} \int_{0}^{\infty} t^{s-1} d t
$$

which is not convergent for any $s \in \mathbb{C}$. Therefore we modify the definition (1.2) as follows.

Definition 4.5. For $k_{1}, \ldots, k_{r} \in \mathbb{Z}_{\geq 0}$ with $\left(k_{1}, \ldots, k_{r}\right) \neq(0, \ldots, 0)$, define

$$
\widetilde{\xi}\left(-k_{1}, \ldots,-k_{r} ; s\right)=\frac{1}{\Gamma(s)} \int_{0}^{\infty} t^{s-1} \frac{\mathrm{Li}_{-k_{1}, \ldots,-k_{r}}\left(1-e^{t}\right)}{e^{-t}-1} d t
$$

for $s \in \mathbb{C}$ with $\operatorname{Re}(s)>1-r$. In the case $r=1$, denote $\widetilde{\xi}(-k ; s)$ by $\widetilde{\xi}_{-k}(s)$ for $k \geq 1$.

We see from (4.4) and (4.5) that (4.8) is well-defined. Also it is noted that $\widetilde{\xi}\left(k_{1}, \ldots, k_{r} ; s\right)$ cannot be defined by replacing $\left\{-k_{j}\right\}$ by $\left\{k_{j}\right\}$ in (4.8) for $\left(k_{j}\right) \in \mathbb{Z}_{>1}^{r}$.

In a way parallel to deriving Theorem 4.4, we can obtain the following.

Theorem 4.6. For $k_{1}, \ldots, k_{r} \in \mathbb{Z}_{\geq 0}$ with $\left(k_{1}, \ldots, k_{r}\right) \neq(0, \ldots, 0), \widetilde{\xi}\left(-k_{1}, \ldots,-k_{r} ; s\right)$ can be analytically continued to an entire function on the whole complex plane, and satisfies

$$
\widetilde{\xi}\left(-k_{1}, \ldots,-k_{r} ;-m\right)=C_{m}^{\left(-k_{1}, \ldots,-k_{r}\right)} \quad\left(m \in \mathbb{Z}_{\geq 0}\right) .
$$

In particular, $\widetilde{\xi}_{-k}(-m)=C_{m}^{(-k)}\left(k \in \mathbb{Z}_{\geq 1}, m \in \mathbb{Z}_{\geq 0}\right)$. 
Next we give certain duality formulas for $B_{n}^{\left(k_{1}, \ldots, k_{r}\right)}$ which is a generalization of (1.6). To state this, we define another type of multi-poly-Bernoulli numbers by

$$
\begin{aligned}
& \sum_{a=0}^{r-1}(-1)^{a}\left(\begin{array}{c}
r-1 \\
a
\end{array}\right) \sum_{l_{1}, \ldots, l_{r} \geq 1} \frac{\prod_{j=1}^{r}\left(1-e^{-\sum_{\nu=j}^{r} x_{\nu}}\right)^{l_{j}-1}}{\left(l_{1}+\cdots+l_{r}-a\right)^{s}} \\
& \quad=\sum_{m_{1}, \ldots, m_{r} \geq 0} \mathfrak{B}_{m_{1}, \ldots, m_{r}}^{(s)} \frac{x_{1}^{m_{1}} \cdots x_{r}^{m_{r}}}{m_{1} ! \cdots m_{r} !}
\end{aligned}
$$

for $s \in \mathbb{C}$. In the case $r=1$, we see that $\mathfrak{B}_{m}^{(k)}=B_{m}^{(k)}$ for $k \in \mathbb{Z}$. Then we obtain the following result which is a kind of the duality formula. In fact, this coincides with (1.6) in the case $r=1$.

Theorem 4.7. For $k_{1}, \ldots, k_{r} \in \mathbb{Z}_{\geq 0}$,

$$
\eta\left(-k_{1}, \ldots,-k_{r} ; s\right)=\mathfrak{B}_{k_{1}, \ldots, k_{r}}^{(s)} .
$$

Therefore, for $m \in \mathbb{Z}_{\geq 0}$,

$$
B_{m}^{\left(-k_{1}, \ldots,-k_{r}\right)}=\mathfrak{B}_{k_{1}, \ldots, k_{r}}^{(-m)} .
$$

Proof. We first prepare the following relation which will be proved in the next section (see Lemma 5.9):

$$
\prod_{j=1}^{r} \frac{e^{\sum_{\nu=j}^{r} x_{\nu}}\left(1-e^{t}\right)}{1-e^{\sum_{\nu=j}^{r} x_{\nu}}\left(1-e^{t}\right)}=\sum_{k_{1}, \ldots, k_{r} \geq 0} \operatorname{Li}_{-k_{1}, \ldots,-k_{r}}\left(1-e^{t}\right) \frac{x_{1}^{k_{1}} \cdots x_{r}^{k_{r}}}{k_{1} ! \cdots k_{r} !}
$$

holds around the origin. Let

$$
\mathcal{F}\left(x_{1}, \ldots, x_{r} ; s\right)=\sum_{k_{1}, \ldots, k_{r} \geq 0} \eta\left(-k_{1}, \ldots,-k_{r} ; s\right) \frac{x_{1}^{k_{1}} \cdots x_{r}^{k_{r}}}{k_{1} ! \cdots k_{r} !} .
$$

As a generalization of [18, Proposition 5], we have from (4.13) that

$$
\begin{aligned}
\mathcal{F}\left(x_{1}, \ldots, x_{r} ; s\right)= & \frac{1}{\Gamma(s)} \int_{0}^{\infty} \frac{t^{s-1}}{1-e^{t}} \prod_{j=1}^{r} \frac{e^{\sum_{\nu=j}^{r} x_{\nu}}\left(1-e^{t}\right)}{1-e^{\sum_{\nu=j}^{r} x_{\nu}}\left(1-e^{t}\right)} d t \\
= & \frac{1}{\Gamma(s)} \int_{0}^{\infty} t^{s-1}\left(1-e^{t}\right)^{r-1} e^{-r t} \prod_{j=1}^{r} \frac{1}{1-e^{-t}\left(1-e^{-\sum_{\nu=j}^{r} x_{\nu}}\right)} d t \\
= & \frac{1}{\Gamma(s)} \sum_{a=0}^{r-1}(-1)^{a}\left(\begin{array}{c}
r-1 \\
a
\end{array}\right) \sum_{m_{1}, \ldots, m_{r} \geq 0} \prod_{j=1}^{r}\left(1-e^{-\sum_{\nu=j}^{r} x_{\nu}}\right)^{m_{j}} \\
& \times \int_{0}^{\infty} t^{s-1} e^{(a-r) t} \prod_{j=1}^{r} e^{-m_{j} t} d t \\
= & \sum_{a=0}^{r-1}(-1)^{a}\left(\begin{array}{c}
r-1 \\
a
\end{array}\right) \sum_{m_{1}, \ldots, m_{r} \geq 0} \frac{\prod_{j=1}^{r}\left(1-e^{-\sum_{\nu=j}^{r} x_{\nu}}\right)^{m_{j}}}{\left(m_{1}+\cdots+m_{r}+r-a\right)^{s}} .
\end{aligned}
$$

Therefore, by (4.10), we obtain (4.11). Further, setting $s=-m$ in (4.11) and using (4.7), we obtain (4.12). 
Remark 4.8. In the case $r=1$, (4.11) implies $\eta_{-k}(s)=B_{k}^{(s)}$. Thus, using Theorem 4.4, we obtain the duality formula (1.6), which is also written as

$$
\eta_{-k}(-m)=\eta_{-m}(-k)
$$

for $k, m \in \mathbb{Z}_{\geq 0}$. This is exactly contrasted with the positive index case (3.11). Furthermore, by the same method, we can show that $\widetilde{\xi}_{-k-1}(-m)=C_{k+1}^{(-m)}$ for $k, m \in \mathbb{Z}_{\geq 0}$. Hence, using Theorem 4.6 in the case $r=1$, we obtain the duality formula (1.7).

Example 4.9. When $r=2$, we can calculate directly from (4.10) that $\mathfrak{B}_{1,0}^{(s)}=3^{-s}-2^{-s}$. On the other hand, as mentioned in Lemma 4.1, we have $\operatorname{Li}_{-1,0}(z)=z^{2} /(1-z)^{3}$. Hence the left-hand side of (1.10) equals

$$
\frac{\mathrm{Li}_{-1,0}\left(1-e^{-t}\right)}{1-e^{-t}}=\frac{1-e^{-t}}{e^{-3 t}}=e^{3 t}-e^{2 t},
$$

hence $B_{m}^{(-1,0)}=3^{m}-2^{m}$. Thus we can verify $B_{m}^{(-1,0)}=\mathfrak{B}_{1,0}^{(-m)}$.

\section{Multi-indexed poly-Bernoulli numbers and duality formulas}

In this section, we define multi-indexed poly-Bernoulli numbers (see Definition 5.1) and prove the duality formula for them, namely a multi-indexed version of (1.6) (see Theorem 5.4).

For this aim, we first recall multiple polylogarithms of $*$-type and of w-type in several variables defined by

$$
\begin{aligned}
& \mathrm{Li}_{s_{1}, \ldots, s_{r}}^{*}\left(z_{1}, \ldots, z_{r}\right)=\sum_{1 \leq m_{1}<\cdots<m_{r}} \frac{z_{1}^{m_{1}} \cdots z_{r}^{m_{r}}}{m_{1}^{s_{1}} m_{2}^{s_{2}} \cdots m_{r}^{s_{r}}}, \\
& \mathrm{Li}_{s_{1}, \ldots, s_{r}}^{\uplus}\left(z_{1}, \ldots, z_{r}\right)=\sum_{1 \leq m_{1}<\cdots<m_{r}} \frac{z_{1}^{m_{1}} z_{2}^{m_{2}-m_{1}} \cdots z_{r}^{m_{r}-m_{r-1}}}{m_{1}^{s_{1}} m_{2}^{s_{2}} \cdots m_{r}^{s_{r}}} \\
& =\sum_{l_{1}, \ldots, l_{r}=1}^{\infty} \frac{z_{1}^{l_{1}} z_{2}^{l_{2}} \cdots z_{r}^{l_{r}}}{l_{1}^{s_{1}}\left(l_{1}+l_{2}\right)^{s_{2}} \cdots\left(l_{1}+\cdots+l_{r}\right)^{s_{r}}}
\end{aligned}
$$

for $s_{1}, \ldots, s_{r} \in \mathbb{C}$ and $z_{1}, \cdots, z_{r} \in \mathbb{C}$ with $\left|z_{j}\right| \leq 1(1 \leq j \leq r)$ (see, for example, [13]). The symbols $*$ and $\boldsymbol{w}$ are derived from the harmonic product and the shuffle product in the theory of multiple zeta values. In fact, Arakawa and the first-named author defined the two types of multiple $L$-values $L^{*}\left(k_{1}, \ldots, k_{r} ; f_{1}, \ldots, f_{r}\right)$ of $*$-type and $L^{ш}\left(k_{1}, \ldots, k_{r} ; f_{1}, \ldots, f_{r}\right)$ of - -type associated to periodic functions $\left\{f_{j}\right\}$ (see [5]), defined by replacing $\left\{z_{j}^{m}\right\}$ by $\left\{f_{j}(m)\right\}$ and setting $\left(s_{j}\right)=\left(k_{j}\right) \in \mathbb{Z}_{\geq 1}^{r}$ on the right-hand sides of (5.1) and (5.2) for $\left(k_{1}, \ldots, k_{r}\right) \in \mathbb{Z}_{\geq 1}^{r}$. Note that

$$
\mathrm{Li}_{s_{1}, \ldots, s_{r}}^{*}\left(z_{1}, \ldots, z_{r}\right)=\mathrm{Li}_{s_{1}, \ldots, s_{r}}^{\mathrm{w}}\left(\prod_{j=1}^{r} z_{j}, \prod_{j=2}^{r} z_{j}, \ldots, z_{r-1} z_{r}, z_{r}\right) .
$$

Definition 5.1 (Multi-indexed poly-Bernoulli numbers). For $s_{1}, \ldots, s_{r} \in \mathbb{C}$ and $d \in\{1,2, \ldots, r\}$, the multi-indexed poly-Bernoulli numbers $\left\{B_{m_{1}, \ldots, m_{r}}^{\left(s_{1}, s_{2}, \ldots, s_{r}\right),(d)}\right\}$ are defined by

$$
F\left(x_{1}, \ldots, x_{r} ; s_{1}, \ldots, s_{r} ; d\right)=\frac{\operatorname{Li}_{s_{1}, \cdots, s_{r}}^{\omega}\left(1-e^{-\sum_{\nu=1}^{r} x_{\nu}}, \ldots, 1-e^{-x_{r-1}-x_{r}}, 1-e^{-x_{r}}\right)}{\prod_{j=1}^{d}\left(1-e^{-\sum_{\nu=j}^{r} x_{\nu}}\right)}
$$




$$
\begin{aligned}
& \left(=\sum_{l_{1}, \ldots, l_{r}=1}^{\infty} \frac{\prod_{j=1}^{r}\left(1-e^{-\sum_{\nu=j}^{r} x_{\nu}}\right)^{l_{j}-\delta_{j}(d)}}{\prod_{j=1}^{r}\left(\sum_{\nu=1}^{j} l_{\nu}\right)^{s_{j}}}\right) \\
& =\sum_{m_{1}, \ldots, m_{r}=0}^{\infty} B_{m_{1}, \ldots, m_{r}}^{\left(s_{1}, \ldots, s_{r}\right),(d)} \frac{x_{1}^{m_{1}} \cdots x_{r}^{m_{r}}}{m_{1} ! \cdots m_{r} !}
\end{aligned}
$$

where $\delta_{j}(d)=1(j \leq d),=0(j>d)$.

Remark 5.2. Note that $\operatorname{Li}_{k_{1}, \ldots, k_{r}}^{\uplus}(z, \ldots, z)=\operatorname{Li}_{k_{1}, \ldots, k_{r}}(z)$ defined by (1.9). Suppose $x_{1}=\cdots=$ $x_{r-1}=0$ and $\left(s_{j}\right)=\left(k_{j}\right) \in \mathbb{Z}^{r}$ in (5.4). We immediately see that if $d=1$ then

$$
B_{0, \ldots, 0, m}^{\left(k_{1}, \ldots, k_{r}\right),(1)}=B_{m}^{\left(k_{1}, \ldots, k_{r}\right)} \quad\left(m \in \mathbb{Z}_{\geq 0}\right)
$$

(see (1.6)), and if $d=r$ then

$$
B_{0, \ldots, 0, m}^{\left(k_{1}, \ldots, k_{r}\right),(r)}=\mathbb{B}_{m}^{\left(k_{1}, \ldots, k_{r}\right)} \quad\left(m \in \mathbb{Z}_{\geq 0}\right)
$$

(see $(1.8))$.

Remark 5.3. Let

$$
\Lambda_{r}=\left\{\left(x_{1}, \ldots, x_{r}\right) \in \mathbb{C}^{r}|| 1-e^{-\sum_{\nu=j}^{r} x_{\nu}} \mid<1(1 \leq j \leq r)\right\} .
$$

Then we can see that

$$
\operatorname{Li}_{s_{1}, \cdots, s_{r}}^{\mathrm{w}}\left(1-e^{-\sum_{\nu=1}^{r} x_{\nu}}, \ldots, 1-e^{-x_{r-1}-x_{r}}, 1-e^{-x_{r}}\right) \quad\left(s_{1}, \ldots, s_{r} \in \mathbb{C}\right)
$$

is absolutely convergent for $\left(x_{j}\right) \in \Lambda_{r}$. Also $F\left(x_{1}, \ldots, x_{r} ; s_{1}, \ldots, s_{r} ; d\right)$ is absolutely convergent in the region $\Lambda_{r} \times \mathbb{C}^{r}$, so is holomorphic. Hence $B_{m_{1}, \ldots, m_{r}}^{\left(s_{1}, \ldots, s_{r}\right),(d)}$ is an entire function, because

$$
B_{m_{1}, \ldots, m_{r}}^{\left(s_{1}, \ldots, s_{r}\right),(d)}=\left.\left(\frac{\partial}{\partial x_{1}}\right)^{m_{1}} \cdots\left(\frac{\partial}{\partial x_{r}}\right)^{m_{r}} F\left(x_{1}, \ldots, x_{r} ; s_{1}, \ldots, s_{r} ; d\right)\right|_{\left(x_{1}, \ldots, x_{r}\right)=(0, \ldots, 0)}
$$

is holomorphic for all $\left(s_{1}, \ldots, s_{r}\right) \in \mathbb{C}^{r}$.

In the preceding section, we gave a certain duality formula for $B_{m}^{\left(k_{1}, \ldots, k_{r}\right)}$ (see Theorem 4.7). By the similar method, we can prove certain duality formulas for $B_{m_{1}, \ldots, m_{r}}^{\left(k_{1}, \ldots, k_{r}\right),(d)}$, though they may be complicated. Hence, in the rest of this section, we will consider the case $d=r$. For emphasis, we denote $B_{m_{1}, \ldots, m_{r}}^{\left(s_{1}, \ldots, s_{r}\right),(r)}$ by $\mathbb{B}_{m_{1}, \ldots, m_{r}}^{\left(s_{1}, \ldots, s_{r}\right)}$. Note that $\delta_{j}(r)=1$ for any $j$. With this notation, we prove the following duality formulas.

Theorem 5.4. For $m_{1}, \ldots, m_{r}, k_{1}, \ldots, k_{r} \in \mathbb{Z}_{\geq 0}$,

$$
\mathbb{B}_{m_{1}, \ldots, m_{r}}^{\left(-k_{1}, \ldots,-k_{r}\right)}=\mathbb{B}_{k_{1}, \ldots, k_{r}}^{\left(-m_{1}, \ldots,-m_{r}\right)} .
$$

Now we aim to prove this theorem. First we generalize Lemma 4.1 as follows.

Lemma 5.5. For $k_{1}, \ldots, k_{r} \in \mathbb{Z}_{\geq 0}$, there exists a polynomial $\widetilde{P}\left(x_{1}, \ldots, x_{r} ; k_{1}, \ldots, k_{r}\right) \in \mathbb{Z}\left[x_{1}, \ldots, x_{r}\right]$ such that

$$
\mathrm{Li}_{-k_{1}, \ldots,-k_{r}}^{*}\left(z_{1}, \ldots, z_{r}\right)=\frac{\widetilde{P}\left(\prod_{j=1}^{r} z_{j}, \prod_{j=2}^{r} z_{j}, \ldots, z_{r-1} z_{r}, z_{r} ; k_{1}, \ldots, k_{r}\right)}{\prod_{j=1}^{r}\left(1-\prod_{\nu=j}^{r} z_{\nu}\right)^{\sum_{\nu=j}^{r} k_{\nu}+1}},
$$




$$
\begin{aligned}
& \operatorname{deg}_{x_{j}} \widetilde{P}\left(x_{1}, \ldots, x_{r} ; k_{1}, \ldots, k_{r}\right) \leq \sum_{\nu=j}^{r} k_{\nu}+1, \\
& \left(x_{1} \cdots x_{r}\right) \mid \widetilde{P}\left(x_{1}, \ldots, x_{r} ; k_{1}, \ldots, k_{r}\right) .
\end{aligned}
$$

Set $y_{j}=\prod_{\nu=j}^{r} z_{\nu}(1 \leq j \leq r)$. Then (5.7) implies

$$
\operatorname{Li}_{-k_{1}, \ldots,-k_{r}}^{\mathrm{w}}\left(y_{1}, \ldots, y_{r}\right)=\frac{\widetilde{P}\left(y_{1}, \cdots, y_{r} ; k_{1}, \ldots, k_{r}\right)}{\prod_{j=1}^{r}\left(1-y_{j}\right)^{\sum_{\nu=j}^{r} k_{\nu}+1}} .
$$

Proof. In order to prove this lemma, we have only to use the same method as in Lemma 4.1 by induction on $r$. Since the case of $r=1$ is proven, we consider the case of $r \geq 2$. Further, when $K=k_{1}+\cdots+k_{r}=0$, it is easy to have the assertion. Hence we think about a general case $K=k_{1}+\cdots+k_{r}(\geq 1)$. When $k_{r}=0$, we have

$$
\begin{aligned}
\mathrm{Li}_{-k_{1}, \ldots,-k_{r}}^{*}\left(z_{1}, \ldots, z_{r}\right) & =\frac{z_{r}}{1-z_{r}} \mathrm{Li}_{-k_{1}, \ldots,-k_{r-1}}^{*}\left(z_{1}, \ldots, z_{r-2}, z_{r-1} z_{r}\right) \\
& =\frac{z_{r}}{1-z_{r}} \frac{\widetilde{P}\left(\prod_{j=1}^{r} z_{j}, \ldots, z_{r-1} z_{r} ; k_{1}, \ldots, k_{r-1}\right)}{\prod_{j=1}^{r-1}\left(1-\prod_{\nu=j}^{r} z_{j}\right)^{\sum_{\nu=j}^{r} k_{\nu}+1}} .
\end{aligned}
$$

Therefore, setting $\widetilde{P}\left(x_{1}, \cdots, x_{r} ; k_{1}, \ldots, k_{r-1}, 0\right)=x_{r} \widetilde{P}\left(x_{1}, \ldots, x_{r-1} ; k_{1}, \ldots, k_{r-1}\right)$, we can verify (5.7)-(5.9) .

Next we consider the case $k_{r} \geq 1$. For $k \in \mathbb{Z}_{\geq 0}$, we inductively define a subset $\left\{c_{j, \nu}^{(k)}\right\}_{0 \leq j, \nu \leq k+1}$ of $\mathbb{Z}$ by

$$
\frac{d}{d z}\left(\sum_{m>l} m^{k} z^{m}\right)=\frac{1}{(1-z)^{k+2}} \sum_{j=0}^{k+1} \sum_{\nu=0}^{k+1} c_{j, \nu}^{(k)} l^{\nu} z^{l+j}
$$

In fact, by

$$
\frac{d}{d z}\left(\sum_{m>l} z^{m}\right)=\frac{1}{(1-z)^{2}}\left(z^{l}+l z^{l}-l z^{l+1}\right)
$$

and

$$
\sum_{m>l} m^{k} z^{m}=z \frac{d}{d z}\left(\sum_{m>l} m^{k-1} z^{m}\right) \quad(k \geq 1),
$$

we can determine $\left\{c_{j, \nu}^{(k)}\right\}$ by (5.11). Using this notation, we have

$$
\begin{aligned}
& \mathrm{Li}_{-k_{1}, \ldots,-k_{r}}^{*}\left(z_{1}, \ldots, z_{r}\right)=z_{r} \frac{d}{d z_{r}} \mathrm{Li}_{-k_{1}, \ldots,-k_{r}+1}^{*}\left(z_{1}, \ldots, z_{r}\right) \\
& =z_{r} \sum_{m_{1}<\cdots<m_{r-1}} m_{1}^{k_{1}} \cdots m_{r-1}^{k_{r-1}} z_{1}^{m_{1}} \cdots z_{r-1}^{m_{r-1}} \frac{\sum_{j=0}^{k_{r}} \sum_{\nu=0}^{k_{r}} c_{j, \nu}^{\left(k_{r}-1\right)} m_{r-1}^{\nu} z_{r}^{m_{r-1}+j}}{\left(1-z_{r}\right)^{k_{r}+1}} \\
& =\frac{1}{\left(1-z_{r}\right)^{k_{r}+1}} \sum_{j=0}^{k_{r}} \sum_{\nu=0}^{k_{r}} c_{j, \nu}^{\left(k_{r}-1\right)} z_{r}^{j+1} \sum_{m_{1}<\cdots<m_{r-1}} m_{1}^{k_{1}} \cdots m_{r-1}^{k_{r-1}+\nu} z_{1}^{m_{1}} \cdots\left(z_{r-1} z_{r}\right)^{m_{r-1}} .
\end{aligned}
$$

By the induction hypothesis in the case $r-1$, this is equal to

$$
\frac{1}{\left(1-z_{r}\right)^{k_{r}+1}} \sum_{j=0}^{k_{r}} \sum_{\nu=0}^{k_{r}} c_{j, \nu}^{\left(k_{r}-1\right)} z_{r}^{j+1} \frac{\widetilde{P}\left(\prod_{j=1}^{r-1} z_{j}, \ldots, z_{r-1} z_{r} ; k_{1}, \ldots, k_{r-2}, k_{r-1}+\nu\right)}{\prod_{j=1}^{r-2}\left(1-\prod_{\nu=j}^{r} z_{j}\right)^{\sum_{\nu=j}^{r} k_{\nu}+1}\left(1-z_{r-1} z_{r}\right)^{k_{r-1}+\nu+1}} .
$$


Therefore we set

$$
\begin{aligned}
& \widetilde{P}\left(x_{1}, \cdots, x_{r} ; k_{1}, \ldots, k_{r}\right) \\
& =\sum_{j=0}^{k_{r}} \sum_{\nu=0}^{k_{r}} c_{j, \nu}^{\left(k_{r}-1\right)} x_{r}^{j+1}\left(1-x_{r-1}\right)^{k_{r}-\nu} \widetilde{P}\left(x_{1}, \cdots, x_{r-1} ; k_{1}, \ldots, k_{r-2}, k_{r-1}+\nu\right) .
\end{aligned}
$$

Then this satisfies (5.7)-(5.9). This completes the proof.

From this result, we can reach the following definition.

Definition 5.6. For $k_{1}, \ldots, k_{r} \in \mathbb{Z}_{\geq 0}$, define

$$
\begin{gathered}
\eta\left(-k_{1}, \ldots,-k_{r} ; s_{1}, \ldots, s_{r}\right)=\frac{1}{\prod_{j=1}^{r} \Gamma\left(s_{j}\right)} \int_{0}^{\infty} \cdots \int_{0}^{\infty} \prod_{j=1}^{r} t_{j}^{s_{j}-1} \\
\times \frac{\mathrm{Li}_{-k_{1}, \ldots,-k_{r}}^{\omega_{1}}\left(1-e^{\sum_{\nu=1}^{r} t_{\nu}}, \ldots, 1-e^{t_{r-1}+t_{r}}, 1-e^{t_{r}}\right)}{\prod_{j=1}^{r}\left(1-e^{\sum_{\nu=j}^{r} t_{\nu}}\right)} \prod_{j=1}^{r} d t_{j}
\end{gathered}
$$

for $s_{1}, \ldots, s_{r} \in \mathbb{C}$ with $\operatorname{Re}\left(s_{j}\right)>0(1 \leq j \leq r)$.

Lemma 5.5 ensures that the integral on the right-hand side of (5.12) is absolutely convergent for $\operatorname{Re}\left(s_{j}\right)>0$. By the same method as in the proof of Theorem 2.3 for $\eta\left(k_{1}, \ldots, k_{r} ; s\right)$, we can similarly obtain the following.

Theorem 5.7. For $k_{1}, \ldots, k_{r} \in \mathbb{Z}_{\geq 0}, \eta\left(-k_{1}, \ldots,-k_{r} ; s_{1}, \ldots, s_{r}\right)$ can be analytically continued to an entire function on the whole complex space, and satisfies

$$
\eta\left(-k_{1}, \ldots,-k_{r} ;-m_{1}, \ldots,-m_{r}\right)=\mathbb{B}_{m_{1}, \ldots, m_{r}}^{\left(-k_{1}, \ldots,-k_{r}\right)} \quad\left(m_{1}, \ldots, m_{r} \in \mathbb{Z}_{\geq 0}\right) .
$$

Proof. As in the proof of Theorem 2.3, let

$$
\begin{aligned}
& H\left(-k_{1}, \ldots,-k_{r} ; s_{1}, \ldots, s_{r}\right) \\
& =\int_{\mathrm{C}^{r}} \prod_{j=1}^{r} t_{j}^{s_{j}-1} \frac{\mathrm{Li}_{-k_{1}, \ldots,-k_{r}}^{\mathrm{w}}\left(1-e^{\sum_{\nu=1}^{r} t_{\nu}}, \ldots, 1-e^{t_{r}}\right)}{\prod_{j=1}^{r}\left(1-e^{\sum_{\nu=j}^{r} t_{\nu}}\right)} \prod_{j=1}^{r} d t_{j} \\
& =\prod_{j=1}^{r}\left(e^{2 \pi i s_{j}}-1\right) \int_{\varepsilon}^{\infty} \cdots \int_{\varepsilon}^{\infty} \prod_{j=1}^{r} t_{j}^{s_{j}-1} \frac{\mathrm{Li}_{-k_{1}, \ldots,-k_{r}}^{\mathrm{m}}\left(1-e^{\sum_{\nu=1}^{r} t_{\nu}}, \ldots, 1-e^{t_{r}}\right)}{\prod_{j=1}^{r}\left(1-e^{\sum_{\nu=j}^{r} t_{\nu}}\right)} \prod_{j=1}^{r} d t_{j} \\
& \quad+\int_{C_{\varepsilon}^{r}} \prod_{j=1}^{r} t_{j}^{s_{j}-1} \frac{\mathrm{Li}_{-k_{1}, \ldots,-k_{r}}^{\mathrm{m}}\left(1-e^{\sum_{\nu=1}^{r} t_{\nu}}, \ldots, 1-e^{t_{r}}\right)}{\prod_{j=1}^{r}\left(1-e^{\sum_{\nu=j}^{r} t_{\nu}}\right)} \prod_{j=1}^{r} d t_{j},
\end{aligned}
$$

where $\mathrm{C}^{r}$ is the direct product of the contour $\mathcal{C}$ defined before. Note that the integrand on the second member has no singularity on $\mathfrak{C}^{r}$. It follows from Lemma 5.5 that $H\left(-k_{1}, \ldots,-k_{r} ; s_{1}, \ldots, s_{r}\right)$ is absolutely convergent for any $\left(s_{j}\right) \in \mathbb{C}^{r}$, namely is entire. Suppose $\operatorname{Re}\left(s_{j}\right)>0$ for each $j$, the second integral tends to 0 as $\varepsilon \rightarrow 0$. Hence

$$
\eta\left(-k_{1}, \ldots,-k_{r} ; s_{1}, \ldots, s_{r}\right)=\frac{1}{\prod_{j=1}^{r}\left(e^{2 \pi i s_{j}}-1\right) \Gamma\left(s_{j}\right)} H\left(-k_{1}, \ldots,-k_{r} ; s_{1}, \ldots, s_{r}\right),
$$

which can be analytically continued to $\mathbb{C}^{r}$. Also, setting $\left(s_{1}, \ldots, s_{r}\right)=\left(-m_{1}, \ldots,-m_{r}\right) \in \mathbb{Z}_{\leq 0}^{r}$ in (5.14), we obtain (5.13) from (5.4). This completes the proof. 
Next we directly construct the generating function of $\eta\left(-k_{1}, \ldots,-k_{r} ; s_{1}, \ldots, s_{r}\right)$. We prepare the following two lemmas which we consider when $\left(x_{j}\right)$ is in $\Lambda_{r}$ defined by (5.5).

Lemma 5.8. For $\left(s_{j}\right) \in \mathbb{C}^{r}$ with $\operatorname{Re}\left(s_{j}\right)>0(1 \leq j \leq r)$,

$$
\begin{aligned}
& F\left(x_{1}, \ldots, x_{r} ; s_{1}, \ldots, s_{r} ; r\right) \\
& =\frac{1}{\prod_{j=1}^{r} \Gamma\left(s_{j}\right)} \int_{0}^{\infty} \cdots \int_{0}^{\infty} \prod_{j=1}^{r}\left\{t_{j}^{s_{j}-1} \frac{e^{\sum_{\nu=j}^{r} x_{\nu}}}{1-e^{\sum_{\nu=j}^{r} x_{\nu}}\left(1-e^{\sum_{\nu=j}^{r} t_{\nu}}\right)}\right\} \prod_{j=1}^{r} d t_{j} .
\end{aligned}
$$

Proof. Substituting $n^{-s}=(1 / \Gamma(s)) \int_{0}^{\infty} t^{s-1} e^{-n t} d t$ into the second member of (5.4), we have

$$
\begin{aligned}
F\left(\left\{x_{j}\right\} ;\left\{s_{j}\right\} ; r\right) & =\sum_{l_{1}, \ldots, l_{r}=1}^{\infty} \prod_{j=1}^{r}\left(1-e^{-\sum_{\nu=j}^{r} x_{\nu}}\right)^{l_{j}-1} \frac{1}{\prod_{j=1}^{r} \Gamma\left(s_{j}\right)} \\
& \times \int_{0}^{\infty} \cdots \int_{0}^{\infty} \prod_{j=1}^{r}\left\{t_{j}^{s_{j}-1} \exp \left(-\left(\sum_{\nu=1}^{j} l_{\nu}\right) t_{j}\right)\right\} \prod_{j=1}^{r} d t_{j} .
\end{aligned}
$$

We see that the integrand on the right-hand side can be rewritten as

$$
\prod_{j=1}^{r} t_{j}^{s_{j}-1} \prod_{j=1}^{r} \exp \left(-l_{j}\left(\sum_{\nu=j}^{r} t_{\nu}\right)\right) \text {. }
$$

Hence we have

$$
\begin{aligned}
F\left(\left\{x_{j}\right\} ;\left\{s_{j}\right\} ; r\right) & =\frac{1}{\prod_{j=1}^{r} \Gamma\left(s_{j}\right)\left(1-e^{-\sum_{\nu=j}^{r} x_{\nu}}\right)} \int_{0}^{\infty} \cdots \int_{0}^{\infty} \prod_{j=1}^{r} t_{j}^{s_{j}-1} \\
& \times \sum_{l_{1}, \ldots, l_{r}=1}^{\infty} \prod_{j=1}^{r}\left(1-e^{-\sum_{\nu=j}^{r} x_{\nu}}\right)^{l_{j}} e^{-l_{j}\left(\sum_{\nu=j}^{r} t_{\nu}\right)} \prod_{j=1}^{r} d t_{j} \\
& =\frac{1}{\prod_{j=1}^{r} \Gamma\left(s_{j}\right)} \int_{0}^{\infty} \cdots \int_{0}^{\infty} \prod_{j=1}^{r} t_{j}^{s_{j}-1} \frac{e^{-\sum_{\nu=j}^{r} t_{\nu}}}{1-\left(1-e^{-\sum_{\nu=j}^{r} x_{\nu}}\right) e^{-\sum_{\nu=j}^{r} t_{\nu}}} \prod_{j=1}^{r} d t_{j} \\
& =\frac{1}{\prod_{j=1}^{r} \Gamma\left(s_{j}\right)} \int_{0}^{\infty} \cdots \int_{0}^{\infty} \prod_{j=1}^{r} t_{j}^{s_{j}-1} \frac{e^{\sum_{\nu=j}^{r} x_{\nu}}}{1-e^{\sum_{\nu=j}^{r} x_{\nu}}\left(1-e^{\sum_{\nu=j}^{r} t_{\nu}}\right)} \prod_{j=1}^{r} d t_{j} .
\end{aligned}
$$

This completes the proof.

Lemma 5.9. Let $z_{1}, \ldots, z_{r} \in \mathbb{C}$ and assume that $\left|z_{j}\right|(1 \leq j \leq r)$ are sufficiently small. Then

$$
\prod_{j=1}^{r} \frac{z_{j} e^{\sum_{\nu=j}^{r} x_{\nu}}}{1-z_{j} e^{\sum_{\nu=j}^{r} x_{\nu}}}=\sum_{k_{1}, \ldots, k_{r}=0}^{\infty} \operatorname{Li}_{-k_{1}, \ldots,-k_{r}}^{\omega_{1}}\left(z_{1}, \ldots, z_{r}\right) \frac{x_{1}^{k_{1}} \cdots x_{r}^{k_{r}}}{k_{1} ! \cdots k_{r} !} .
$$

Set $z_{j}=1-e^{\sum_{\nu=j}^{r} t_{\nu}}(1 \leq j \leq r)$ for $\left(t_{j}\right) \in \Lambda_{r}$. Then

$$
\begin{aligned}
& \prod_{j=1}^{r} \frac{e^{\sum_{\nu=j}^{r} x_{\nu}}\left(1-e^{\sum_{\nu=j}^{r} t_{\nu}}\right)}{1-e^{\sum_{\nu=j}^{r} x_{\nu}}\left(1-e^{\sum_{\nu=j}^{r} t_{\nu}}\right)} \\
& \quad=\sum_{k_{1}, \ldots, k_{r}=0}^{\infty} \mathrm{Li}_{-k_{1}, \ldots,-k_{r}}^{\mathrm{\omega}}\left(1-e^{\sum_{\nu=1}^{r} t_{\nu}}, \ldots, 1-e^{t_{r}}\right) \frac{x_{1}^{k_{1}} \cdots x_{r}^{k_{r}}}{k_{1} ! \cdots k_{r} !} .
\end{aligned}
$$

In particular, the case $t_{1}=\cdots=t_{r-1}=0$ and $t_{r}=t$ implies (4.13). 
Proof. We have only to prove (5.16). Actually we have

$$
\begin{aligned}
& \sum_{k_{1}, \ldots, k_{r}=0}^{\infty} \mathrm{Li}_{-k_{1}, \ldots,-k_{r}}^{\mathrm{w}}\left(z_{1}, \ldots, z_{r}\right) \frac{x_{1}^{k_{1}} \cdots x_{r}^{k_{r}}}{k_{1} ! \cdots k_{r} !} \\
= & \sum_{k_{1}, \ldots, k_{r}=0}^{\infty} \sum_{m_{1}, \ldots, m_{r}=1}^{\infty} \prod_{j=1}^{r} \frac{\left(\left(\sum_{\mu=1}^{j} m_{\mu}\right) x_{j}\right)^{k_{j}}}{k_{j} !} z^{m_{j}} \\
= & \sum_{m_{1}, \ldots, m_{r}=1}^{\infty} \prod_{j=1}^{r} z_{j}^{m_{j}} \prod_{\mu=1}^{j} e^{m_{\mu} x_{j}} \\
= & \sum_{m_{1}, \ldots, m_{r}=1}^{\infty} \prod_{j=1}^{r}\left(z_{j} e^{\sum_{\nu=j}^{r} x_{\nu}}\right)^{m_{j}}=\prod_{j=1}^{r} \frac{z_{j} e^{\sum_{\nu=j}^{r} x_{\nu}}}{1-z_{j} e^{\sum_{\nu=j}^{r} x_{\nu}} .}
\end{aligned}
$$

Thus we have the assertion.

Using these lemmas, we obtain the following.

Theorem 5.10. For $k_{1}, \ldots, k_{r} \in \mathbb{Z}_{\geq 0}$,

$$
\eta\left(-k_{1}, \ldots,-k_{r} ; s_{1}, \ldots, s_{r}\right)=\mathbb{B}_{k_{1}, \ldots, k_{r}}^{\left(s_{1}, \ldots, s_{r}\right)} .
$$

Proof. By Lemmas 5.8 and 5.9, we have

$$
\begin{aligned}
& F\left(x_{1}, \ldots, x_{r} ; s_{1}, \ldots, s_{r} ; r\right) \\
& =\frac{1}{\prod_{j=1}^{r} \Gamma\left(s_{j}\right)} \int_{0}^{\infty} \cdots \int_{0}^{\infty} \prod_{j=1}^{r}\left\{t_{j}^{s_{j}-1} \frac{e^{\sum_{\nu=j}^{r} x_{\nu}}}{1-e^{\sum_{\nu=j}^{r} x_{\nu}}\left(1-e^{\sum_{\nu=j}^{r} t_{\nu}}\right)}\right\} \prod_{j=1}^{r} d t_{j} \\
& =\frac{1}{\prod_{j=1}^{r} \Gamma\left(s_{j}\right)} \sum_{k_{1}, \ldots, k_{r}=0}^{\infty}\left\{\int_{0}^{\infty} \cdots \int_{0}^{\infty} \prod_{j=1}^{r} t_{j}^{s_{j}-1}\right. \\
& \left.\quad \times \frac{\mathrm{Li}_{-k_{1}, \ldots,-k_{r}}^{\uplus_{1}}\left(1-e^{\sum_{\nu=1}^{r} t_{\nu}}, \ldots, 1-e^{t_{r}}\right)}{\prod_{j=1}^{r}\left(1-e^{\sum_{\nu=j}^{r} t_{\nu}}\right)} \prod_{j=1}^{r} d t_{j}\right\} \frac{x_{1}^{k_{1}} \cdots x_{r}^{k_{r}}}{k_{1} ! \cdots k_{r} !}
\end{aligned}
$$

for $\operatorname{Re}\left(s_{j}\right)>0(1 \leq j \leq r)$. Combining (5.4), (5.12) and (5.19), we obtain (5.18) for $\operatorname{Re}\left(s_{j}\right)>0$ $(1 \leq j \leq r)$, hence for all $\left(s_{j}\right) \in \mathbb{C}$, because both sides of (5.18) are entire functions (see Remark $5.3)$.

Proof of Theorem 5.4. Setting $\left(s_{1}, \ldots, s_{r}\right)=\left(-m_{1}, \ldots,-m_{r}\right)$ in (5.18), we obtain (5.6) from (5.13). This completes the proof of Theorem 5.4.

Example 5.11. We can easily see that

$$
\mathrm{Li}_{-1,0}^{\mathrm{w}}\left(z_{1}, z_{2}\right)=\frac{z_{1} z_{2}}{\left(1-z_{1}\right)^{2}\left(1-z_{2}\right)}, \quad \operatorname{Li}_{0,-1}^{\mathrm{w}}\left(z_{1}, z_{2}\right)=\frac{z_{1} z_{2}\left(2-z_{1}-z_{2}\right)}{\left(1-z_{1}\right)^{2}\left(1-z_{2}\right)^{2}} .
$$

Hence we have

$$
\mathbb{B}_{m, n}^{(-1,0)}=2^{m} 3^{n}, \quad \mathbb{B}_{m, n}^{(0,-1)}=\left(2^{m}+1\right) 3^{n} \quad\left(m, n \in \mathbb{Z}_{\geq 0}\right) .
$$

Therefore $\mathbb{B}_{0,1}^{(-1,0)}=\mathbb{B}_{1,0}^{(0,-1)}=3$. Similarly we obtain, for example,

$$
\mathbb{B}_{1,0}^{(-1,-2)}=\mathbb{B}_{1,2}^{(-1,0)}=18, \mathbb{B}_{1,2}^{(-3,-1)}=\mathbb{B}_{3,1}^{(-1,-2)}=1820, \mathbb{B}_{2,2}^{(-2,-1)}=\mathbb{B}_{2,1}^{(-2,-2)}=1958
$$


Remark 5.12. Hamahata and Masubuchi [14, Corollary 10] showed the special case of (5.6), namely

$$
\mathbb{B}_{0, \ldots, 0, m}^{(0, \ldots, 0,-k)}=\mathbb{B}_{0, \ldots, 0, k}^{(0, \ldots, 0,-m)} \quad\left(m, k \in \mathbb{Z}_{\geq 0}\right)
$$

(see Remark 5.2). On the other hand, Theorem 4.7 corresponds to the case $d=1 \neq r$ except for $r=1$ (see Remark 5.2), hence is located in the outside of Theorem 5.4. Therefore, in (4.12), another type of multi-poly-Bernoulli numbers appear.

\section{References}

[1] S. Akiyama, S. Egami and Y. Tanigawa, Analytic continuation of multiple zeta functions and their values at non-positive integers, Acta Arith., 98 (2001), 107-116.

[2] K. Aomoto, Special values of hyperlogarithms and linear difference schemes, Illinois J. Math., 34-2 (1990), 191-216.

[3] T. Arakawa, T. Ibukiyama and M. Kaneko, Bernoulli Numbers and Zeta Functions, Springer, Tokyo, 2014.

[4] T. Arakawa and M. Kaneko, Multiple zeta values, poly-Bernoulli numbers, and related zeta functions, Nagoya Math. J., 153 (1999), 189-209.

[5] T. Arakawa and M. Kaneko, On multiple $L$-values, J. Math. Soc. Japan, 56 (2004), 967991.

[6] A. Bayad and Y. Hamahata, Polylogarithms and poly-Bernoulli polynomials, Kyushu J. Math., 65 (2011), 15-24.

[7] A. Bayad and Y. Hamahata, Arakawa-Kaneko L-functions and generalized poly-Bernoulli polynomials, J. Number Theory, 131 (2011), 1020-1036.

[8] A. Bayad and Y. Hamahata, Multiple polylogarithms and multi-poly-Bernoulli polynomials, Funct. Approx. Comment. Math., 46 (2012), 45-61.

[9] C. Brewbaker, A combinatorial interpretation of the Poly-Bernoulli numbers and two Fermat analogues, Integers, 8 (2008), $\sharp$ A02.

[10] M. -A. Coppo and B. Candelpergher, The Arakawa-Kaneko zeta function, Ramanujan J., 22 (2010), 153-162.

[11] V. G. Drinfel'd, On quasitriangular quasi-Hopf algebras and a group closely connected with $\operatorname{Gal}(\overline{\mathbb{Q}} / \mathbb{Q})$, Leningrad Math. J. 2 (1991), 829-860.

[12] H. Furusho, $p$-adic multiple zeta values. I. $p$-adic multiple polylogarithms and the $p$-adic KZ equation, Invent. Math., 155 (2004), 253-286.

[13] A. B. Goncharov, Multiple polylogarithms, cyclotomy, and modular complexes, Math. Res. Lett., 5 (1998), 497-516.

[14] Y. Hamahata and H. Masubuchi, Special multi-poly-Bernoulli numbers, J. Integer Seq., 10 (2007), Article 07.4.1. 
[15] Y. Hamahata and H. Masubuchi, Recurrence formulae for multi-poly-Bernoulli numbers, Integers, 7 (2007), $\sharp$ A46.11.

[16] M. Hoffman, Multiple harmonic series, Pacific J. Math., 152 (1992), 275-290.

[17] K. Imatomi, Multi-poly-Bernoulli-star numbers and finite multiple zeta-star values, Integers, 14 (2014), A51.

[18] K. Imatomi, M. Kaneko and E. Takeda, Multi-poly-Bernoulli numbers and finite multiple zeta values, J. Integer Sequences, 17 (2014), Article 14.4.5.

[19] K. Ihara, M. Kaneko and D. Zagier, Derivation and double shuffle relations for multiple zeta values, Compositio Math., 142 (2006), 307-338.

[20] M. Kaneko, Poly-Bernoulli numbers, J. Théor. Nombres Bordeaux, 9 (1997), 199-206.

[21] M. Kaneko, Poly-Bernoulli numbers and related zeta functions, Algebraic and Analytic Aspects of Zeta Functions and L-functions, MSJ Mem., 21, pp.73-85, Math. Soc. Japan, Tokyo, 2010.

[22] M. Kaneko and M. Sakata, Notes on height one multiple zeta values, in preparation.

[23] T. Q. T. Le and J. Murakami, Kontsevich's integral for the Homfly polynomial and relations between values of multiple zeta functions, Topology Appl., 62 (1995), 193-206.

[24] Y. Ohno, A generalization of the duality and sum formulas on the multiple zeta values, J. Number Theory, 74 (1999), 39-43.

[25] S. Oi, Gauss hypergeometric functions, multiple polylogarithms, and multiple zeta values, Publ. Res. Inst. Mat. Sci. 45 (2009), 981-1009.

[26] J. Okuda and K. Ueno, Relations for multiple zeta values and Mellin transforms of multiple polylogarithms, Publ. Res. Inst. Math. Sci., 40 (2004), 537-564.

[27] J. Okuda and K. Ueno, The sum formula for multiple zeta values and connection problem of the formal Knizhnik-Zamolodchikov equation, Zeta Functions, Topology and Quantum Physics, Dev. Math., 14, Springer, New York, (2005), 145-70.

[28] Y. Sasaki, On generalized poly-Bernoulli numbers and related $L$-functions, J. Number Theory, 132 (2012), 156-170.

[29] G. Shimura, Elementary Dirichlet Series and Modular Forms, Springer Monographs in Mathematics, Springer, New York, 2007.

[30] L. C. Washington, Introduction to Cyclotomic Fields, Second edition, Graduate Texts in Mathematics 83, Springer-Verlag, New York, 1997.

[31] P. T. Young, Symmetries of Bernoulli polynomial series and Arakawa-Kaneko zeta functions, J. Number Theory, 143 (2014), 142-161. 
M. Kaneko: Faculty of Mathematics, Kyushu University, Motooka 744, Nishi-ku Fukuoka 819-0395, Japan

Tel \& Fax: 092-802-4443

e-mail: mkaneko@math.kyushu-u.ac.jp

H. Tsumura: Department of Mathematics and Information Sciences, Tokyo Metropolitan University, 1-1, Minami-Ohsawa, Hachioji, Tokyo 192-0397 Japan

e-mail: tsumura@tmu.ac.jp 